\title{
Tip Motion-Sensor Signal Relation for a Composite SPM/SPL Cantilever
}

\author{
Dennis Roeser, Stefanie Gutschmidt, Thomas Sattel, and Ivo W. Rangelow
}

\begin{abstract}
An array of microbeams is a promising approach to increase the throughput of scanning probe microscopes and lithography. This concept requires integrated sensors and actuators which enable individual measurement and control. Thus, existing models for single beams need to be reassessed in view of its applicability for arrays, which involve additional physical interactions and a varying geometry along the beam's length. This paper considers a single composite microbeam, which is excited by a thermal actuator and its displacement is measured by a piezoresistive sensor. We derive a model that incorporates the beam's composite structure, varying geometry along its length, its thermal coupling for actuation, and thermoelastic damping. Subsequently, the influence of the beam's geometry on its eigenmodes and frequencies is analyzed in far and close proximity operation to a surface. We observe parametric excitation phenomena of multiple integers of the fundamental excitation frequency, which originates from the geometrical composition of the beam. Furthermore, we observe that the so far constant assumed factor to convert the sensor signal to the beam's displacement depends on the dissipated power within the actuator, as well as on the dynamic behavior of the system, and thus is not constant.

[2015-0208]
\end{abstract}

Index Terms-Arrays, thermal actuation, piezoresistive sensing, microelectromechanical systems (MEMS), thermomechanical coupling.

\section{INTRODUCTION}

$\mathbf{S}$ CANNING probe microscopy (SPM) has been studied for the past few decades and has become a commercially available tool for nanometer metrology. By optically measuring the displacement of the free end of a microbeam, amplitude shifts due to interaction forces can be detected. By controlling the amplitude of oscillation, a positioning stage is used to maintain a constant distance. The motion of this stage gives the coordinates of the sample and the hight information. Using a similar setup, manipulation and patterning of a sample

Manuscript received July 24, 2015; revised September 12, 2015; accepted September 18, 2015. This work was supported by the European Union Seventh Framework Programme FP7/2007-2013 through the Project entitled Single Nanometer Manufacturing for Beyond CMOS Devices under Grant 318804. The work of D. Roeser was supported by the Carl Zeiss Foundation for Ph.D. Scholarships. Subject Editor L. Buchaillot.

D. Roeser and T. Sattel are with the Department of Mechatronics, Ilmenau University of Technology, Ilmenau 98693, Germany (e-mail: dennis.roeser@tu-ilmenau.de; thomas.sattel@tu-ilmenau.de).

S. Gutschmidt is with the Mechanical Engineering Department, University of Canterbury, Christchurch 8140, New Zealand (e-mail: stefanie.gutschmidt@ canterbury.ac.nz).

I. W. Rangelow is with the Department of Microelectronic and Nanoelectronic Systems, Ilmenau University of Technology, Ilmenau 98693, Germany (e-mail: ivo.rangelow@tu-ilmenau.de).

Color versions of one or more of the figures in this paper are available online at http://ieeexplore.iee.org.

Digital Object Identifier 10.1109/JMEMS.2015.2482389 can be done. For instance, the Fowler-Nordheim scanning probe lithography (FN-SPL) is a very promising candidate offering routinely resolutions below $10 \mathrm{~nm}$, an overlay accuracy below $1 \mathrm{~nm}$, and a relatively low equipment costs [1]. This technology employs very low number of electrons (low exposure dose) with energies just suitable to provide highly localized chemical reactions into the resist. Critical for this process is to keep the distance between the microbeam's free end and the resist covered sample constant, due to the dependency of the electron emission from the tip sample distance. Similar to SPM, the beams free end is measured and the distance is controlled by using a positioning stage. Both methods, SPM and SPL, offer advantages with respect to resolution, range of application (less demands on probe preparation) and capital investment cost, thus, providing the means for future desktop nanofabrication. However, a major drawback of the scanning probe technologies is the limited throughput, rendering the process slow and less applicable for mass production/analysis.

One way to improve the throughput of SPL and SPM is the use of microbeam arrays. Unlike conventional scanning probe technology (where tip deflections are measured with respect to an absolute reference frame), individual tip deflections in the array configuration are measured with respect to a relative reference frame (e.g. using piezoresistive sensors). Accurate measurements and thus the quality of property predictions are highly dependent on the knowledge of the dynamic behaviour of individual resonators [2], [3] as well as their collective dynamic behaviour. Additionally, the distance between tip and sample is set individually per beam by means of an integrated actuator and thus also yields the topographic information as well as the distance to the sample. Typical approaches for the tip motion estimation multiply the sensor signal with a constant factor to gain the displacement of the tip [4]-[6]. A in-depth analysis regarding this estimation as well as a noncontact SPM or SPL operation with a constant distance of such an array has not been achieved to date.

Within this article, a single microbeam of an array is considered, which has a thermal actuator and a piezoresistive sensor, as depicted in Figure 1. This microbeam and the corresponding array have been subjected to intensive experimental investigations, regarding integrated circuit technology, SPM scan process as well as SPL [4], [6]-[10]. To gain a deeper insight in experimentally observed phenomena (e.g. coupling between modes [11], coupling between beams in an array [12], [13]) as well as to achieve a control scheme for maintaining a constant distance for SPL and noncontact SPM processes, a model sets the foundation. Demand for this model is a sufficient 


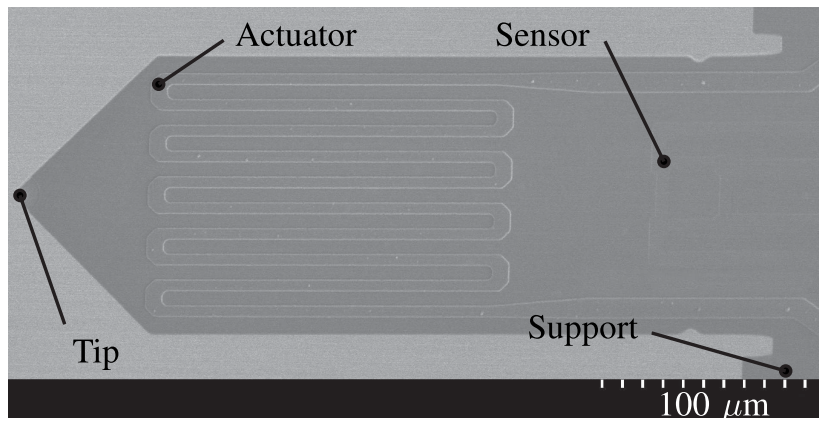

Fig. 1. SEM image of a single microbeam of an array, with integrated thermal actuator and piezoresistive sensor.

prediction of the tip movement based on the mechanical stress at the fixed end, by taking into account the composite structure and thermal coupling of the microbeam. In order to derive a mathematical model of the full array device (including all salient field interactions) in the future, a systematic theoretical investigation of a single microbeam with thermal actuator and piezoresistive sensor (Figure 1) sets the basis of this work.

To date, various modeling approaches, that take into account the coupled electro-thermo-mechanical problem, are based on the static behavior of a bimorph actuator. For instance, Bullen et al. [14] and Watanabe et al. [15] approach a thermally actuated microbeam by means of a deflection curve of a composite beam, including the heat conduction formulation for the static case. Li et al. [16], in contrast, approach the coupled electro-thermo-mechanical problem by means of a nodal analysis, utilizing circuit simulation software to achieve a less computational expensive model. Approaches in view of a dynamical analysis of coupled thermo-mechanical fields focus on the effects of thermal damping in microbeams [17]-[19]. As outlined in these articles, the thermo-elastic damping has a significant influence on the dynamical behavior and depends on the geometrical dimensions of the resonator [17], [18]. They also exhibit an influence in view of a shift in resonant frequency, outnumbering the influence of air damping of the order of $10^{-3}$ [17].

To face the challenges of predicting the motion of the beam's tip, explain the dynamics of beam as well as to set the basis for an array model, we derived a coupled thermoelastic model, based on an Euler-Bernoulli beam, Fourier heat conduction equation and a Lennard-Jones force potential in Section II, summarized in Section II-C. Subsequently, a modal analysis in Section III investigates the influence of the beam's geometry and the interaction forces on eigenmodes and -frequency, with an emphasis on the sensor and tip position. Section III also includes a dynamic analysis, in view of different types of excitation and the prediction of the tip movement based on the mechanical stress at the piezoresistive bridge. Finally, the results are summarized and discussed in Section IV.

\section{MODEL}

The governing equations of motion of the composite structure are based on the thermoelastic constitutive law and

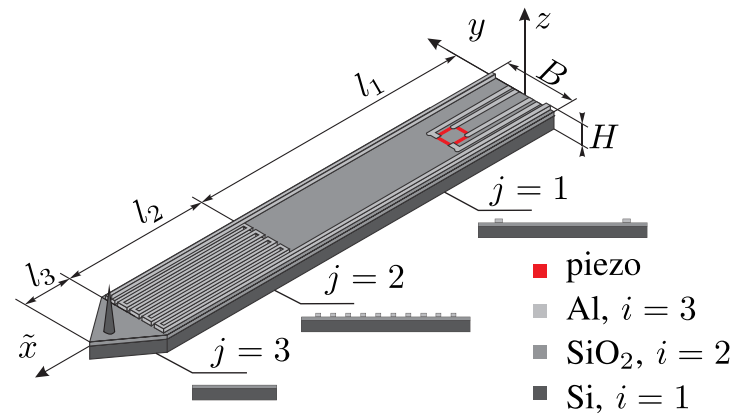

(a)

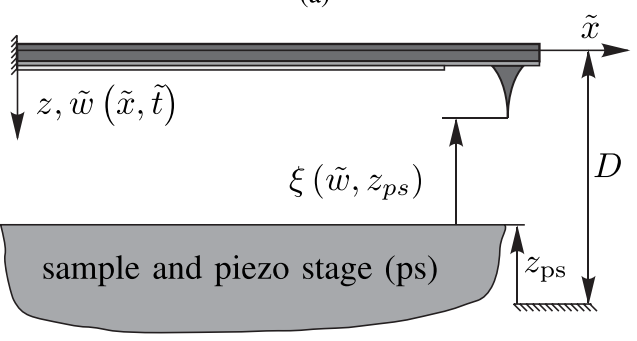

(b)

Fig. 2. Sketches of (a) the cantilever and (b) the tip-sample kinematics.

the Euler-Bernoulli beam theory for transverse vibrations. The actuation mechanism is modelled by the Fourier heat conduction equation. The composite structure of the beam is composed of three layers (index $i$, c.f. Figure 2a): Silicon $(i=1)$, Silicon Dioxide $(i=2)$ and Aluminum $(i=3)$. The Aluminum layer is the thermal actuator and determines the varying cross sections along the beam's length. Accordingly, the beam is divided into three sections (index $j$ ), with a length $l_{j}$ for $j=1,2,3$ (see Figure 2a). The origin of the body fixed coordinate system is at the fixed end of the cantilever (see Figure 2a):

$$
0 \leq \tilde{x} \leq L, \quad-\frac{B}{2} \leq y \leq \frac{B}{2}, \quad-\frac{H}{2} \leq z \leq \frac{H}{2} .
$$

Next to the global coordinate system, additional coordinate systems are introduced for the second and third section, where their origins are shifted with respect to $\tilde{x}$ by $l_{1}$ and $l_{1}+l_{2}$, respectively. The overall thickness and length of the beam are given by $H=\sum_{i} h_{i}$ and $L=\sum_{j} l_{j}$. Variables denoted by tilde are rescaled and made nondimensional in Section II-C, [20]. The cross-sectional area is given by

$$
A_{j i}= \begin{cases}h_{i} B, & j=1,2,3 \wedge i=1,2 ; \\ h_{i} b_{H j} N_{j}, & j=1,2,3 \wedge i=3 ;\end{cases}
$$

where the beam's width is $B$, the width of one actuator wire is $b_{H j}$ and the number of wires per section is $N_{j}$. The beam's displacement $\tilde{w}(\tilde{x}, \tilde{t})$ is positive toward the sample surface and is given by:

$$
\tilde{w}(\tilde{x}, \tilde{t})= \begin{cases}\tilde{w}_{1}(\tilde{x}, \tilde{t}), \quad 0 \leq \tilde{x}<l_{1} ; \\ \tilde{w}_{2}(\tilde{x}, \tilde{t}), \quad l_{1} \leq \tilde{x}<l_{1}+l_{2} ; \\ \tilde{w}_{3}(\tilde{x}, \tilde{t}), \quad l_{1}+l_{2} \leq \tilde{x}<L .\end{cases}
$$

The time varying gap between sample and tip is

$$
\xi\left(\tilde{w}, z_{p s}\right)=D-h_{t}-\tilde{w}\left(L-l_{t}, \tilde{t}\right)-z_{p s}(\tilde{t}),
$$


with $h_{t}, l_{t}$ and $z_{p s}$ being the tip height, distance to tip from the beam's free end and the displacement of the piezoelectric positioning stage, respectively. The initial distance between origin of coordinates and the sample in $z$ direction is $D$ (c.f. Figure 2b).

\section{A. Mechanical System}

Derivations concerning the physics of the mechanical structure are based on Newton's second law and the Duhamel-Neuman law, which is a generalization of Hooke's law to account for the influence of heating [21]. In any arbitrary section $j$ and layer $i$, the mechanical stress for pure bending is given by $\sigma_{\tilde{x} j i}=E_{i} \epsilon_{j}-E_{i} \alpha_{i} \tilde{\theta}_{j}$, with the strain $\epsilon_{j}=\left(z-z_{0 j}\right) / \kappa_{j}$, the curvature of the beam $\kappa_{j}^{-1}$, the coefficient of thermal expansion $\alpha_{i}$ and the temperature difference $\tilde{\theta}_{j}\left(\tilde{x}_{j}, \tilde{t}\right)=T_{j}\left(\tilde{x}_{j}, \tilde{t}\right)-T_{0}$, wherein $T_{j}$ is the absolute temperature and $T_{0}$ represents the initial temperature. The distance to the neutral fibre $z_{0 j}$ in (5) is derived from the equilibrium of forces in $\tilde{x}$ direction, for the case of pure bending. Together with the strain $\epsilon_{j}, z_{0 j}$ and the equilibrium of the moments about the $y$ axis yield the corresponding moment $M_{j}$ in (6) (see also [22], [23]).

$$
\begin{aligned}
z_{0 j}= & \bar{A}_{j}^{-1}\left(\kappa_{j}^{-1} \bar{S}_{\mathrm{y} j}-\overline{A \alpha}_{j} \tilde{\theta}_{j}\right) \kappa_{j}, \\
M_{j}= & E_{1}\left(\bar{I}_{\mathrm{y} j}-\bar{A}_{j}^{-1} \bar{S}_{\mathrm{y} j}^{2}\right) \kappa_{j}^{-1} \\
& +E_{1}\left(\bar{A}_{j}^{-1} \bar{S}_{\mathrm{y} j} \overline{A \alpha}_{j}-\bar{S}_{\mathrm{y} \alpha}\right) \tilde{\theta}_{j},
\end{aligned}
$$

with the constants

$$
\begin{aligned}
n_{i} & =\frac{E_{i}}{E_{1}}, \quad \bar{A}_{j}=\sum_{i} n_{i} A_{j i}, \\
\overline{A \alpha}_{j} & =\sum_{i} n_{i} \alpha_{i} A_{j i}, \quad \bar{S}_{\mathrm{y} j}=\sum_{i} n_{i} S_{\mathrm{y} j i}, \\
\bar{S}_{\mathrm{y}} \alpha_{j} & =\sum_{i} n_{i} \alpha_{i} S_{\mathrm{y} j i}, \quad \bar{I}_{\mathrm{y} j}=\sum_{i} n_{i} I_{\mathrm{y} j i} .
\end{aligned}
$$

Young's modulus, weighting factor and the cross sectional area are given by $E_{i}, n_{i}$ and $A_{j i}$, respectively. The first moment of area is $S_{y j i}=z_{\mathrm{CG} i} A_{j i}$ and the distance to each layer's center of gravity $z_{\mathrm{CG} i}$ as well as the second moment of area $I_{\mathrm{y} j i}$ are given by

$$
\begin{aligned}
& z_{\mathrm{CGi}}= \frac{h_{i}}{2}-\frac{H}{2}+\sum_{\bar{i}=1}^{i-1} h_{\bar{i}}, \\
& I_{\mathrm{y} j i}= \begin{cases}\left(\frac{h_{i}^{3}}{12}+z_{C G i}^{2} h_{i}\right) B, & i=1,2 ; \\
\left(\frac{h_{i}^{3}}{12}+z_{C G i}^{2} h_{i}\right) b_{H j} N_{j}, & i=3 .\end{cases}
\end{aligned}
$$

The governing equation for an infinitesimal beam element is derived using Newton's second law under Bernoulli assumptions. An approximation for the curvature of the beam $\kappa_{j}^{-1} \approx-\tilde{w}_{j, \tilde{x} \tilde{x}}$, is assumed, with $\tilde{w}_{j, \tilde{x}} \ll 1[24]$ :

$$
\begin{aligned}
\mu_{j} \tilde{w}_{j, \tilde{t} \tilde{t}}= & E_{1} \underbrace{\left(\bar{A}_{j}^{-1} \bar{S}_{\mathrm{y} j} \overline{A \alpha}_{j}-\bar{S}_{\mathrm{y} \alpha}\right) \tilde{\theta}_{j, \tilde{x} \tilde{x}}}_{\text {thermal coupling and excitation }} \\
& -\underbrace{d_{j} \tilde{w}_{j, \tilde{t}}-E_{1}\left(\bar{I}_{\mathrm{y} j}-\bar{A}_{j}^{-1} \bar{S}_{\mathrm{y} j}^{2}\right) \tilde{w}_{j, \tilde{x} \tilde{x} \tilde{x} \tilde{x}}}_{\text {viscous damping and elastic restoring force }} \\
& +\underbrace{\bar{A}_{j}^{-1} F_{\mathrm{TS}}\left(\tilde{w}_{j}\right)}_{\text {interaction forces }} .
\end{aligned}
$$

Partial derivatives with respect to spatial coordinate and time are denoted with ()$_{\tilde{x}}$ and ()$_{\tilde{x}}$, respectively. The mass per unit length is given by $\mu_{j}=\sum_{i} \rho_{j i} A_{j i}$ and $F_{T S}$ is the tip sample interaction force. Accordingly, the associated boundary and continuity conditions (for $j=1,2$ ) are

$$
\begin{aligned}
\tilde{w}_{1}(0, \tilde{t})=0, & \tilde{w}_{j}\left(l_{j}, \tilde{t}\right)=\tilde{w}_{j+1}(0, \tilde{t}), \\
\tilde{w}_{1, \tilde{x}}(0, \tilde{t})=0, & \tilde{w}_{j, \tilde{x}}\left(l_{j}, \tilde{t}\right)=\tilde{w}_{j+1, \tilde{x}}(0, \tilde{t}), \\
M_{3}(L, \tilde{t})=0, & M_{j}\left(l_{j}, \tilde{t}\right)=M_{j+1}(0, \tilde{t}), \\
M_{3, \tilde{x}}(L, \tilde{t})=0, & M_{j, \tilde{x}}\left(l_{j}, \tilde{t}\right)=M_{j+1, \tilde{x}}(0, \tilde{t}) .
\end{aligned}
$$

Boundary (right column) and continuity conditions (left column) in (10) from top to bottom represent the conditions of the beam at either end with respect to its displacement, angle, moment and shear force, respectively.

Equation (9) describes the motion of the composite microbeam shown in Figure 2a. The elastic restoring term also depends on the first moment of area $S_{y}$, since the beam axis and the axis of the center of gravity along the beams length are not identically in each section. Note that a different prestress for each layer can easily be included through the stress/strain relation stated at the beginning of this section. Even though a prestress is induced in each layer due to the fabrication process, it mainly affects the systems behavior with a constant offset in displacement and sensor signal and is thus not considered in this article. The interaction force in (9) is derived from a Lennard Jones potential for a spherical tip and a flat sample pair and is given by

$$
\begin{aligned}
F_{\mathrm{TS}}\left(\tilde{w}, z_{\mathrm{ps}}\right)= & -\frac{A_{h} R_{t} \sigma^{6}}{180 \xi\left(\tilde{w}, z_{\mathrm{ps}}\right)^{8}} \delta\left(\tilde{x}_{3}+l_{3}-l_{t}\right) \\
& +\frac{A_{h} R_{t}}{6 \xi\left(\tilde{w}, z_{\mathrm{ps}}\right)^{2}} \delta\left(\tilde{x}_{3}+l_{3}-l_{t}\right),
\end{aligned}
$$

wherein $\sigma$ is a typical atomic diameter for a sphere tip and flat sample, $A_{h}$ is the Hamaker constant and $R_{t}$ is the radius of the tip [25], [26]. This force is included into the equation of motion (9) by means of the Dirac delta function $\delta\left(\tilde{x}_{3}+l_{3}-l_{t}\right)$, and spatially limited to act only at the beam's tip. The thermal coupling term represents the actuation of the beam by means of Joule heating and the bimetal effect. Additionally, the coupling to the thermal system leads to an intrinsic thermal damping, as can be seen in the next Section II-B.

\section{B. Thermal System and Actuation}

The thermal system is based on the first and second law of thermodynamics as well as on Fourier's one dimensional heat 
conduction equation in $\tilde{x}$ direction, for an isotropic material with temperature independent mechanical material properties. Due to the large volume of the chip to which the cantilever is attached, the fixed end of the beam is assumed to be isothermal. Considering the small cross-sectional area and low pressure conditions, we further assume the free end to be adiabatic. With a temperature difference $\theta_{j}$ being small compared to the initial temperature $T_{0}$, the governing equation can be written as (see [21], [27])

$$
\begin{aligned}
& \left({\overline{c_{v} \rho A}}_{j}+T_{0} E_{1} \overline{A \alpha}_{j}^{2} \bar{A}_{j}^{-1}\right) \tilde{\theta}_{j, \tilde{t}} \\
& =\underbrace{\bar{k}_{t} A_{j} \tilde{\theta}_{j, \tilde{x} \tilde{x}}}_{\text {heat flux }}-\underbrace{T_{0} E_{1}\left(\bar{A}_{j}^{-1} \bar{S}_{y j} \overline{A \alpha}_{j}-\bar{S}_{y}{ }_{j}\right) \tilde{w}_{j, \tilde{x} \tilde{x} \tilde{t}}}_{\text {mechanical coupling, thermal damping }} \\
& +\underbrace{\bar{A}_{j}\left(g_{\mathrm{a} j}(i)+g_{\mathrm{s} j}\left(U_{\mathrm{s}}(\tilde{t})\right)\right)}_{\text {internal heat generation }},
\end{aligned}
$$

and the coefficients are

$$
{\overline{k_{t}}}_{j}=\sum_{i} k_{t j i} A_{j i}, \quad{\overline{c_{v} \rho A}}_{j}=\sum_{i} c_{v j i} \rho_{j i} A_{j i} .
$$

The thermal boundary (left) and continuity (right) conditions (for $j=1,2$ ) are given by

$$
\begin{aligned}
\tilde{\theta}_{1}(0, \tilde{t}) & =0, \quad \tilde{\theta}_{j}\left(l_{j}, \tilde{t}\right)=\tilde{\theta}_{j+1}(0, \tilde{t}), \\
\tilde{\theta}_{3, \tilde{x}}(L, \tilde{t}) & =0, \quad k_{t j} \tilde{\theta}_{j, \tilde{x}}\left(l_{j}, \tilde{t}\right)=k_{t j+1} \tilde{\theta}_{j+1, \tilde{x}}(0, \tilde{t}),
\end{aligned}
$$

where (13a) are the temperature and (13b) the heat flux boundary and continuity conditions.

Equation (12) describes the heat transfer within the composite beam, of each section $j$. The mechanical coupling represents an internal damping mechanism of the mechanical system, wherein the beam motion leads to a heat generation. As outlined by Guo and Rogerson [17], this thermal damping is of a similar order than that of the viscous damping in air and is the dominant damping mechanism in vacuum operation. An internal heat is generated by two volumetric sources, the power supply of the sensor $g_{\mathrm{s} j}(\tilde{t})$ and the power from the excitation signal $g_{\mathrm{a} j}(i(\tilde{t}))$, given by

$$
\begin{aligned}
g_{s j}(\tilde{t}) & =\frac{U_{\mathrm{s}}(\tilde{t})^{2}}{R_{\mathrm{s}} V_{\mathrm{s}}}, \quad j=1 ; \\
g_{\mathrm{a} j}(\tilde{t}) & =\frac{\rho_{\mathrm{e}}}{A_{j 3}^{2}}\left(1+\alpha_{\mathrm{e}} \tilde{\theta}\right) i(\tilde{t})^{2}, \quad j=1,2 .
\end{aligned}
$$

In which $U_{\mathrm{S}}(\tilde{t})$ is the sensor bridge supply Voltage, $R_{\mathrm{S}}$ and $V_{\mathrm{S}}$ are the constant assumed overall resistance and volume of the piezoresistive sensor elements, respectively. The thermal actuator (resistivity $\rho_{\mathrm{e}}$, temperature coefficient of the resistivity $\alpha_{\mathrm{e}}$ ) dissipates heat within the first two sections as well as within the third layer $(j=1,2 ; i=3)$, with an applied current of

$$
i(\tilde{t})=i_{\mathrm{dc}}(\tilde{t})+i_{\mathrm{ac}} \sin (\Omega \tilde{t}),
$$

where $i_{\mathrm{dc}}(\tilde{t})$ is the direct current, $i_{\mathrm{ac}}$ the alternating current and $\Omega$ the excitation frequency. Equation (14) includes a temperature dependant resistivity, which leads to a time dependent and periodic coefficient in (12). In case of a $i_{\mathrm{dc}}(\tilde{t})$ used as a control factor, this coefficient can also be aperiodic.

\section{Coupled System}

In the following, derived models of Section (II-A) and (II-B) will be discretized in view of the analysis in Section III. Together with governing coupled equations of motion of the microbeam the output signal of the pirzoresistive sensor is presented. To rescale (9) and (12), the following parameters are introduced:

$$
\begin{aligned}
x_{j} & =\frac{\tilde{x}_{j}}{L}, \quad w_{j}=\frac{\tilde{w}_{j}}{H}, \quad t=\omega_{s 1} \tilde{t}, \quad \theta_{j}=\frac{\tilde{\theta}_{j}}{\theta_{0}}, \\
\omega_{s}^{2} & =\frac{\left(\bar{I}_{y 1} \bar{A}-\bar{S}_{y, 1}^{2}\right) E_{1}}{L^{4} \mu_{1} \bar{A}_{1}} .
\end{aligned}
$$

The reference temperature difference $\theta_{0}=T_{R}-T_{0}$ is based on a reference temperature $T_{R}$ of $303.15 \mathrm{~K}$. For the spatial discretization, the following mixed Ritz approach is applied to (9) and (12)

$$
\begin{aligned}
w_{j}\left(x_{j}, t\right) & =\sum_{n=1}^{N} \Phi_{w j n}\left(x_{j}\right) q_{w j n}(t)=\boldsymbol{\Phi}_{w}^{T} \boldsymbol{q}_{w}, \\
\theta_{j}\left(x_{j}, t\right) & =\sum_{n=1}^{N} \Phi_{\theta j n}\left(x_{j}\right) q_{\theta j n}(t)=\boldsymbol{\Phi}_{\theta}^{T} \boldsymbol{q}_{\theta},
\end{aligned}
$$

where $\Phi_{w j n}$ and $\Phi_{\theta j n}$ are comparison functions, $q_{w j n}$ and $q_{\theta j n}$ are time varying amplitudes for the $n$-th mode of the mechanical and thermal system, respectively. Bold symbols represent a vector or matrix, where the vectors $\boldsymbol{\Phi}_{w / \theta}$ and $\boldsymbol{q}_{w / \theta}$ are column vectors containing the summands from (17) and (29) from $n=1$ until $N$. The comparison functions are based on the undamped and homogeneous equations (9) and (12), with $F_{\mathrm{TS}}=0$ (see Appendix A). To discretize the equations of motion, approach (17) and (29) are introduced to (9) and (12) and a Galerkin method is applied, with multiplying mechanical and thermal equation with $\boldsymbol{\Phi}_{w}$ and $\boldsymbol{\Phi}_{\theta}$, respectively, and integrate both equations with respect to the $x$ direction. Accordingly, the equations of motion of the coupled system can be written to

$$
\begin{aligned}
\boldsymbol{J}_{1} \ddot{\boldsymbol{q}}_{w} & =-\boldsymbol{J}_{2} \dot{\boldsymbol{q}}_{w}-\boldsymbol{J}_{3} \boldsymbol{q}_{w}+\boldsymbol{J}_{4} \boldsymbol{q}_{\theta}+\boldsymbol{F}_{\mathrm{TS}}, \\
\boldsymbol{J}_{5} \dot{\boldsymbol{q}}_{\theta} & =\left(\boldsymbol{J}_{6}+\boldsymbol{J}_{7} i^{2}\right) \boldsymbol{q}_{\theta}-\boldsymbol{J}_{8} \dot{\boldsymbol{q}}_{w}+\boldsymbol{J}_{9} i^{2}+\boldsymbol{J}_{10} U_{\mathrm{s}}^{2} .
\end{aligned}
$$

$\boldsymbol{J}_{k}$ are constants in (19) and can be found in Appendix B. The system of ordinary differential equations (19) represents the motion of the microbeam with a composite structure and thermal actuation. The coupling between mechanical and thermal system is mainly defined by $\boldsymbol{J}_{\boldsymbol{4}}$ and $\boldsymbol{J}_{\boldsymbol{\delta}}$, which represent the thermal actuation and thermal damping of the system. The current $i$ (see (15)) is the excitation signal, which also leads to time dependent coefficients and thus an additional parametric excitation for the thermal subsystem. The bridge circuit of the piezoresistive sensor elements (see Figure $2 \mathrm{a}$ with the piezo marked in red) is introduced with a constant supply voltage $U_{\mathrm{s}}$ and the following voltage drop as an output signal [10]:

$$
U_{\mathrm{b}}=2 \frac{\Delta R}{R} U_{\mathrm{s}}, \quad \frac{\Delta R}{R}=\frac{\pi_{1} L}{l_{\mathrm{s}}} \int_{l_{\mathrm{s} 1} / L}^{l_{\mathrm{s} 2} / L} \sigma_{j i} \mathrm{~d} x,
$$

wherein $\pi_{1}$ is the longitudinal piezoresistive coefficients, $\Delta R / R$ the relative resistance change, $l_{\mathrm{s}}=l_{\mathrm{s} 2}-l_{\mathrm{s} 1}$ the length 
of the piezoresistor and the mechanical stress $\sigma_{j i}$ in $\tilde{x}$ direction of the beam, given by

$$
\begin{aligned}
\sigma_{j i}= & \left(E_{i} \bar{S}_{y j} \bar{A}_{j}^{-1}-E_{i} z_{k}\right) H l^{-2} \boldsymbol{\Phi}_{w, x x}^{T} \boldsymbol{q}_{w} \\
& +\left(E_{i} \overline{A \alpha}_{j}-E_{i} \bar{A}_{j} \alpha_{i}\right) \bar{A}_{j} \theta_{0} \boldsymbol{\Phi}_{\theta}^{T} \boldsymbol{q}_{\boldsymbol{\theta}} .
\end{aligned}
$$

The nondimensional tip-sample force acting at the beam's tip in (19) is given by

$$
\begin{aligned}
\boldsymbol{F}_{\mathrm{TS}}= & -\chi_{7}\left(\xi_{0}-H \hat{\boldsymbol{\Phi}}_{w}^{T} \boldsymbol{q}_{w}-z_{p s}\right)^{-8} \\
& +\boldsymbol{\chi}_{8}\left(\xi_{0}-H \hat{\boldsymbol{\Phi}}_{w}^{T}-z_{\mathrm{ps}}\right)^{-2},
\end{aligned}
$$

with $\xi_{0}=D-h_{t}$, as depicted in the kinematic sketch in Figure 2b. The shape functions $\hat{\boldsymbol{\Phi}}_{w}^{T}$ in (22) represents the beam's displacement at the tip position $\left(\hat{\boldsymbol{\Phi}}_{w}^{T}=\boldsymbol{\Phi}_{w}^{T}\left(1-l_{t} / L\right)\right)$.

Thus, the coupled thermo-mechanical system (19) describes the motions of a composite beam depicted in Figure 1 and Figure 2, with a varying cross section, thermal excitation through resistive heating and the bimetal effect. It is applicable to an AFM noncontact operation in a low pressure environment, for small angles $\tilde{w}_{j, \tilde{x}} \ll 1$ as well as in case of isotropic layers, with no convection at the free end and an isothermal fixed end. Additionally, the temperature change is assumed to be small compared to the the surrounding temperature, $\tilde{\theta} \ll T_{0}$. Note that the sign of the curvature due to a temperature difference needs to be determined for each section based on the sign of the thermal coupling and excitation term in (9).

\section{ANALYSIS}

The analysis presented in this section is focused on special sets of properties of the considered system, including the varying geometry, the thermal actuation as well as the sensing mechanism and its relation to the displacement of the beam's free end. These aspects are viewed in terms of their influence on the eigenmodes and eigenfrequencies, their implications on possible internal resonances and the equilibrium solution as well as on the prediction of the tip displacement, based on the mechanical stress at the clamped end for different types of excitation.

\section{A. Modal Analysis}

In this section the influence of different cross-sectional areas along the beam's length and composition of layers on the eigenmodes and -frequencies is analysed. Therefore, an isotropic Euler-Bernoulli beam (using volume weighted parameters) and a constant cross section is compared to the proposed model (see Section II).

Figure 3 shows the first three eigenfunctions $\Phi_{\mathrm{B} n}$ of a Euler-Bernoulli model for an isotropic beam over the dimensionless coordinate $x$ (line style: solid $\Phi_{\mathrm{B} 1}$; dotted $\Phi_{\mathrm{B} 2}$; dashed $\left.\Phi_{\mathrm{B} 3}\right)$. The shaded area represents the deviation between the comparison functions $\Phi_{w n}$ of the composite, sectional model compared to the eigenfunctions $\Phi_{\mathrm{B} n}$ of the Euler-Bernoulli beam model for a variation of the geometry of the thermal actuator. Varied parameters are the length of section two $\left(l_{2} ; j=2\right)$ as well as the height of the aluminum

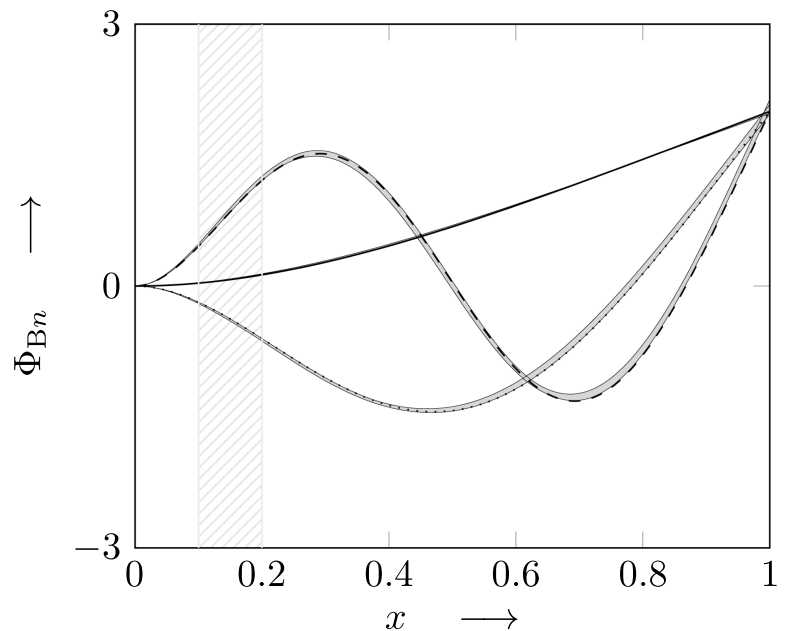

Fig. 3. Eigenfunctions $\Phi_{\mathrm{B} n}$ for a variation of $l_{2}, b_{2}$ and $h_{3}$ at $F_{T S}=0$; solid lines $\Phi_{\mathrm{B} 1}$; dotted lines $\Phi_{\mathrm{B} 2}$; dashed lines $\Phi_{\mathrm{B} 3}$; shaded regions: deviation between $\Phi_{\mathrm{B} n}$ and $\Phi_{w n}$ (parameter range according to Table I).

layer $\left(h_{3} ; j=1,2 ; i=3\right)$, according to the parameters in Table II and Table I in Appendix C. The overall length $L$ and height $H$ of the the beam are held constant. The hatched area represents the position of the piezoresistive sensor. As can be seen in Figure 3, the influence of a varied stiffness increases with higher eigenmodes and manifests itself in a shift of the nodes as well as a shift of the local maxima and minima of the eigenmode. These effects mainly occur in regions of varied stiffness values, at the location of the actuator. Note that the eigenmode at the free end of the beam has a larger deviation compared to that of the sensor position, which is increasing for higher modes. The similarity between eigenfunctions $\Phi_{\mathrm{B} n}$ and comparison functions $\Phi_{w n}$ in Figure 3 is high for the parameters used (see Appendix C), but strongly depend on the overall geometry of the beam $(L, B, H)$ and the position of the actuator. Considering different parameters can lead to an increased deviation, especially in the areas stated above.

In an SPL/SPM operation with arrays, it is important to set and measure the actual displacement of the beam's free end. For instance, a possible array control scheme for SPM based on the work of Ivanova et al. [9] could work in the following way: Each beam is excited at a fixed frequency $\hat{\Omega}$ with a constant amplitude $i_{\mathrm{ac}}$ (which is set in close proximity to a surface). A change in distance to the sample surface $D$ leads to a change in oscillation amplitude, which is measured by the integrated sensor and compensated by a static displacement of the beam induced by changing $i_{\mathrm{dc}}$ to restore the initial distance $D_{0}$. The feedback for the amplitude control is measured from the bridge signal $U_{\mathrm{b}}$. Information regarding the topography of the sample is included in the evolution of $i_{\mathrm{dc}}$ during the scan as well as from the sensor signal $U_{\mathrm{b}}$. Focusing on the latter, one approach to gain a quantitative displacement is to multiply the sensor voltage with a constant conversion factor [5], [6]. This conversion factor $\left(\kappa_{\mathrm{w} n}\right)$ can be derived from the static response of the discretized equation of motion (19):

$$
\begin{aligned}
\tilde{w}_{\mathrm{sr}}\left(l_{t}, \tilde{t}\right) & =\boldsymbol{J}_{\mathbf{3}}{ }^{-1} \boldsymbol{J}_{\mathbf{4}}\left(\boldsymbol{J}_{\boldsymbol{6}}+\boldsymbol{J}_{7} i^{2}\right)^{-1} \boldsymbol{J}_{\boldsymbol{g}} i^{2} ; \\
\kappa_{\mathrm{w} n} & =\frac{\tilde{w}_{\mathrm{sr}}\left(l_{t}, \tilde{t}\right)}{U_{\mathrm{b}}} .
\end{aligned}
$$




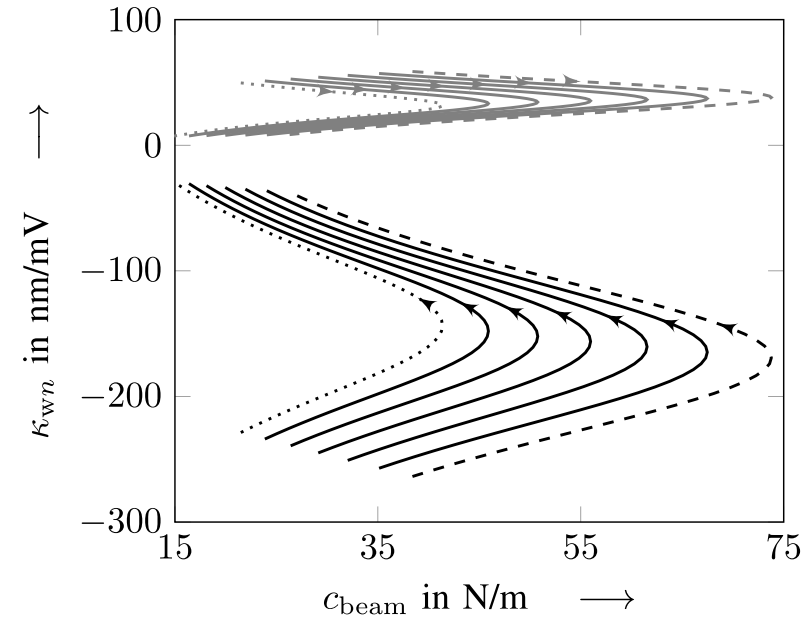

Fig. 4. Conversion factor $\kappa_{\text {wu }}$ vs beam stiffness $c_{\text {beam }}$ for a displacement according to $\Phi_{w 1}$ (black line) and $\Phi_{w 2}$ (gray line) for a variation of $h_{3}$ from $h_{3}=0.05 H$ (dotted line) to $h_{3}=0.2 H$ (dashed line); arrow indicates increase of $l_{2}$ from $l_{2}=0.2 L$ to $l_{2}=0.8 L$.

This approach is similar to a calibration routine, in which the beam is displaced by a constant current $i_{\mathrm{dc} 0}$, the displacement $\tilde{w}_{\text {sr }}\left(l_{t}, \tilde{t}\right)$ is measured by means of an optical sensor and is related to the measured sensor voltage to gain the conversion factor $\kappa_{\mathrm{w} n}$. Thus, $\tilde{w}_{\mathrm{est}}(\tilde{t})=\kappa_{\mathrm{uw}} U_{\mathrm{b}}$ represents the sensor based displacement estimate.

Figure 4 shows the evolution of the sensitivity factor $\kappa_{\mathrm{w} n}$ for a variation of the actuator's length $l_{2}$ and height $h_{3}$. Note that the inverse of the conversion factor is the sensitivity of the sensor, indicating that a small conversion factor is related to a high sensitivity. Especially the first mode shows a strong influence of $l_{2}$ on $\kappa_{\mathrm{w} n}$, yielding different sensitivities for a beam with a constant stiffness $c_{\text {beam }}$. The influence of the actuator's height $h_{3}$ decreases for an increased length $l_{2}$. The second eigenmode of the beam shows a higher sensitivity compared to the first mode and is also less affected by geometrical variations. Thus, for a tip motion estimation in multi frequency SPM different factors for the modes apply. In contrast to a bulk Euler-Bernoulli beam model, the ratio between the sensitivity factors of the two modes is not constant.

Figure 5 illustrates the dependency of the ratio of the second to the first eigenfrequency on the actuator geometry $\left(b_{2}, l_{2}\right.$ and $\left.h_{3}\right)$. The line style represents different heights of the aluminum layer (solid lines $b_{2}=0.5 B$, dashed lines $b_{2}=B$, dotted lines $b_{2}=1.5 B$ ). The corresponding eigenvalues have been obtained solving the eigenvalue problem of (38), based on an isochronic approach (see Appendix A). Numerically, a root finding algorithm based on the bisection and secant method as well as on an inverse quadratic interpolation has been used [28]. In all cases, an increasing thickness of the aluminum layers leads to an increasing influence of a variation of the length of the second section (actuator meander) on the ratio of eigenfrequencies. This dependency is also observed experimentally, with $\omega_{2} / \omega_{1} \approx 6.44$, [11] (circle in Figure 5). Note that a bulk model has a constant frequency ration of $\omega_{2} / \omega_{1} \approx 6.27$ (black solid line).

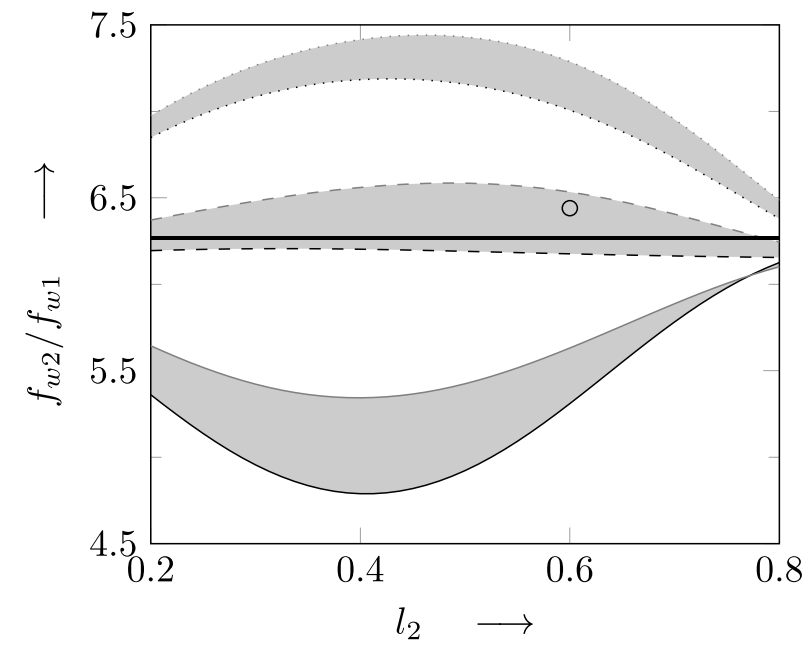

Fig. 5. Frequency ratio $f_{w 2} / f_{w 1}$ for variation of $l_{2}, h_{3}$ and $b_{2}$; thick solid line: Bulk Euler-Bernoulli beam; solid line: $b_{2}=.5 B$; dashed lines $b_{2}=B$; dotted lines $b_{2}=1.5 B$; shaded regions: composite model for varied $h_{3}$; black color: $h_{3}=0.05 H$; gray color: $h_{3}=0.2 H$. The circle indicates experimental results [11].

In addition, the geometric composition of the beam can lead to internal resonances. For values of $b_{2}=0.5 B$ and $b_{2}=1.5 B$ seven-to-one and five-to-one internal resonances occur, respectively. As already shown by Hacker and Gottlieb [29], by considering a nonideal fixed boundary, a geometry variation along the beam's length can also lead to two-to-one internal resonances. These internal resonances can cause unwanted distortion, especially when using multi modes simultaneously. On the other hand, a combined two frequency operation utilizing internal resonance can also lead to an increased speed or sensitivity Hacker and Gottlieb [29].

\section{B. Equilibrium Analysis}

In order to draw conclusions on the occurrence of internal resonances due to the varying stiffness along the beam's length in conjunction with the interaction potential, the system is further analyzed in close proximity to a sample surface. Thus, assuming all time derivatives of $\boldsymbol{q}_{w}$ and $\boldsymbol{q}_{\theta}$ as well as all time varying terms to be equal to zero, the fixed point equation is given by (constants are shown in Appendix B)

$$
\begin{aligned}
\boldsymbol{F}\left(\boldsymbol{q}_{w}, \xi_{0}\right)= & -\boldsymbol{J}_{3} \boldsymbol{q}_{w}-\boldsymbol{J}_{4}\left(\boldsymbol{J}_{6}+\boldsymbol{J}_{7} i_{d c}^{2}\right)^{-1} \boldsymbol{J}_{9} i_{d c}^{2} \\
& +\boldsymbol{\chi}_{7}\left(\xi_{0}-H \hat{\boldsymbol{\Phi}}_{w}^{T} \boldsymbol{q}_{w}-z_{p s}\right)^{-8} \\
& -\boldsymbol{\chi}_{8}\left(\xi_{0}-H \hat{\boldsymbol{\Phi}}_{w}^{T} \boldsymbol{q}_{w}-z_{p s}\right)^{-2} .
\end{aligned}
$$

A Moore-Penrose continuation algorithm [30] is applied to calculated fix points depicted in Figure 6. Here, the line style corresponds to different stiffnesses of the beam, from low stiffness (solid lines, $3.7 \mathrm{~N} / \mathrm{m}$ ) to high stiffness (dashed line $23.8 \mathrm{~N} / \mathrm{m}$ ). Figure 6 shows different bifurcation points, depending on the stiffness of the beam. For a low stiffness beam, two saddle node bifurcations occur (indicated with a circle), with a stable solution above the surface (black circle, lower branch), a stable solution in contact (gray circle, upper branch) and an unstable branch in between. 


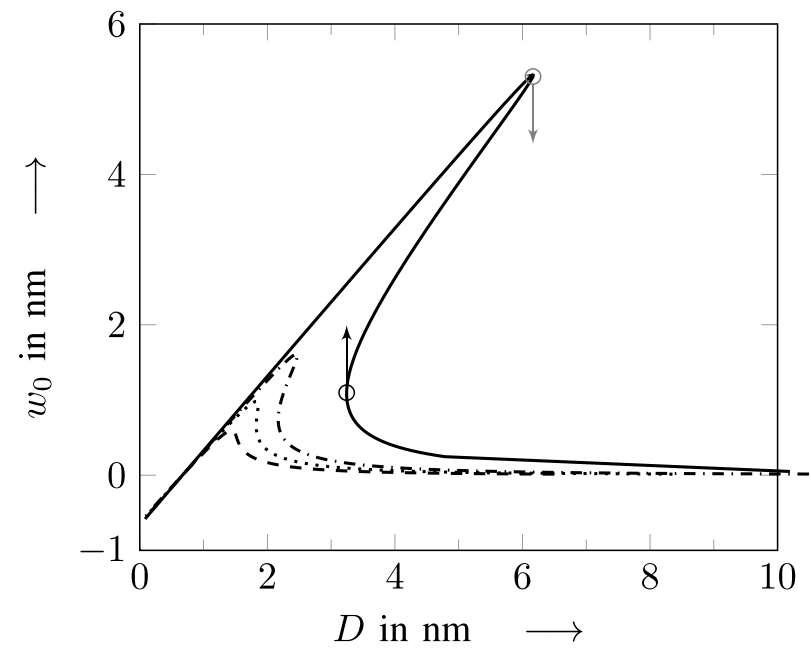

Fig. 6. Equilibrium solutions of (24) in case of beams with varying stiffness; solid line: $3.7 \mathrm{~N} / \mathrm{m}$; dotted line: $7.2 \mathrm{~N} / \mathrm{m}$; dash dotted line: $10 \mathrm{~N} / \mathrm{m}$; dashed line: $23.8 \mathrm{~N} / \mathrm{m}$; circles and arrays instancing bifurcation points and jump direction for approach (black) and retreat (gray) to/from a surface in case of a stiffness of $3.7 \mathrm{~N} / \mathrm{m}$.

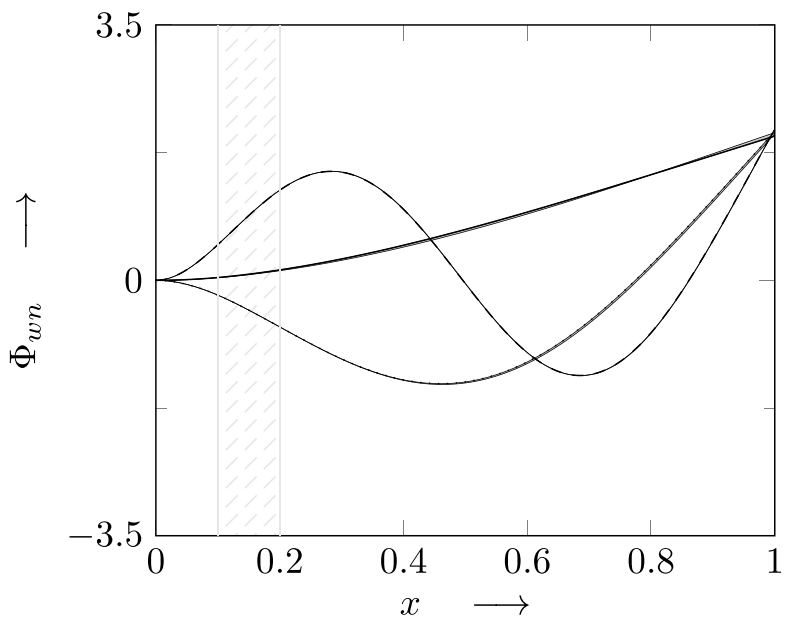

Fig. 7. Comparison functions $\Phi_{w n}$ for a variation of the tip sample interaction force, varying $F_{T S} ; b_{2}, l_{2}, h_{3}$ being constant; solid lines $\Phi_{w 1}$; dotted lines $\Phi_{w 2}$; dashed lines $\Phi_{w 3}$; shaded regions: deviation between the comparison function $\Phi_{w n}$ for $\boldsymbol{F}_{\mathrm{TS}}=\boldsymbol{0}$ and $\boldsymbol{F}_{\mathrm{TS}} \neq \boldsymbol{0}$ (parameter range according to Table I).

This behavior also reassembles the hysteresis observed in SPL/SPM operation when approaching (jump to contact, upward arrow) and retracting (snap of contact, downward arrow) a surface. In contrast, only stable solutions are present for a high stiffness beam, without any bifurcations.

Approaching a surface the interaction forces change from attractive to repulsive. Figure 7 illustrates changes induced to the eigenmode for such an approach. The interaction potential is linearized for each point and the eigenfunctions are calculated with a mixed Ritz approach and a numerical root finding algorithm [28] (see Appendix A). Similar to Figure 3, the line style represents the mode and the shaded area around each line is the deviation from the model with interaction forces compared to the non perturbed model. As can be seen, the forces mainly affect the eigenfunctions for $x>0.2$,

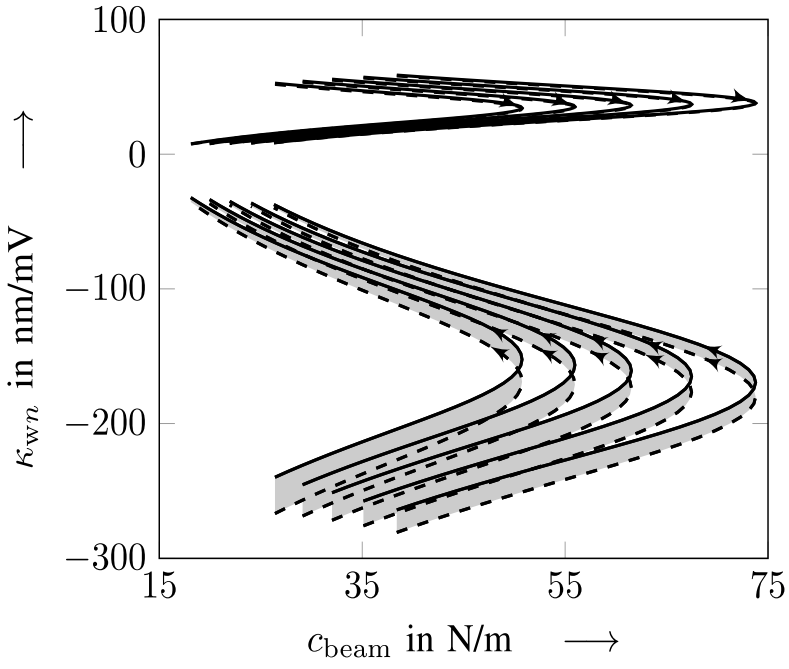

Fig. 8. Conversion factor $\kappa_{\mathrm{wu}}$ over the beam stiffness $c_{\text {beam }}$ for a displacement according to $\Phi_{w 1}$ (black line) and $\Phi_{w 2}$ (gray line) for a variation of $h_{3}$ from $h_{3}=0.05 H$ (dotted line) to $h_{3}=0.2 H$ (dashed line); arrow indicates increase of $l_{2}$ from $l_{2}=0.2 L$ to $l_{2}=0.8 L$; shaded area indicate variation of tip-sample separation from $D=10 \mathrm{~nm}$ (dash dotted line) to $D=0.1 \mathrm{~nm}$ (solid line).

whereas the effect decreases for higher modes. Considering the sensor position at the beam's fixed end (hatched area in Figure 7) the deviation is negligible. Note that the deviation shown also depend on the overall geometry of the beam as well as the tip-sample interaction pair.

Figure 8 illustrates the conversion factor $\kappa_{\mathrm{w} n}$ over the beam stiffness $c_{\text {beam }}$ in case of a variation of the length $l_{2}$, the height $h_{3}$ as well as the distance between tip and sample $D$ (see Figure 2b). The line styles and variation is similar to Figure 4, with the additional shaded area indicating the difference between different tip sample separations, with $D=0.1 \mathrm{~nm}$ (dash dotted line) to $D=10 \mathrm{~nm}$ (solid line). The effect of a variation of $D$ on $\kappa_{\mathrm{w} n}$ is mainly present for the first mode $\left(\Phi_{w 1}\right)$, and increases with a decrease of length $l_{2}$. For $l_{2} \approx 0.45 L$ no variation of $\kappa_{\mathrm{w} n}$ is present for the first mode. As can be seen, the second mode $\left(\Phi_{w 2}\right)$ shows no significant change of $\kappa_{\mathrm{w} n}$ for a variation of $D$.

Corresponding to the fix point solutions in Figure 6, the shift of the eigenvalues is shown in Figure 9, for different distances and sectional compositions. The eigenvalues have been calculated based on (38) in Appendix A, with a interaction force linearized at the corresponding fix point and a numerical root finding algorithm [28]. For a beam with low stiffness, the first eigenvalue has only a real part for the unstable solution branch. Higher eigenvalues exhibit an oval evolution between the two saddle node bifurcations. In case of a high stiffness beam without bifurcations, only hardening and softening can be observed. Figure 9 also illustrates the change in sensitivity to changes in the distance between tip and sample. In case of the first mode, the change in eigenvalue over the distance is very steep close to the bifurcation points, with a rapid decreasing slope for higher modes. The high stiffness beam (dashed lines) already exhibits a lower sensitivity for the first eigenvalue, reducing even further for higher eigenvalues. 


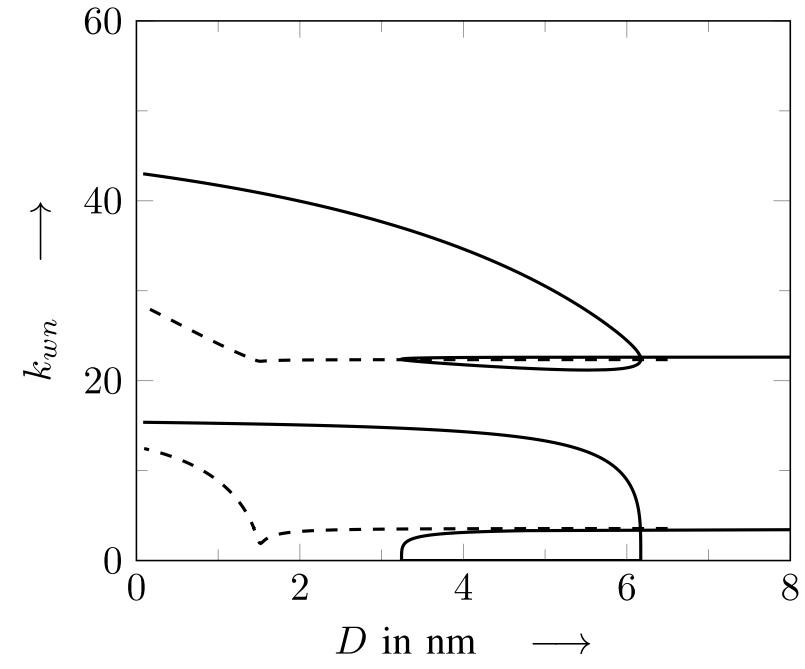

Fig. 9. Eigenvalues $k_{w n}$ for a linearized system at the fix points of (24) in case of beams with varying stiffness; solid line: $3.7 \mathrm{~N} / \mathrm{m}$; dashed line: $23.8 \mathrm{~N} / \mathrm{m}$.

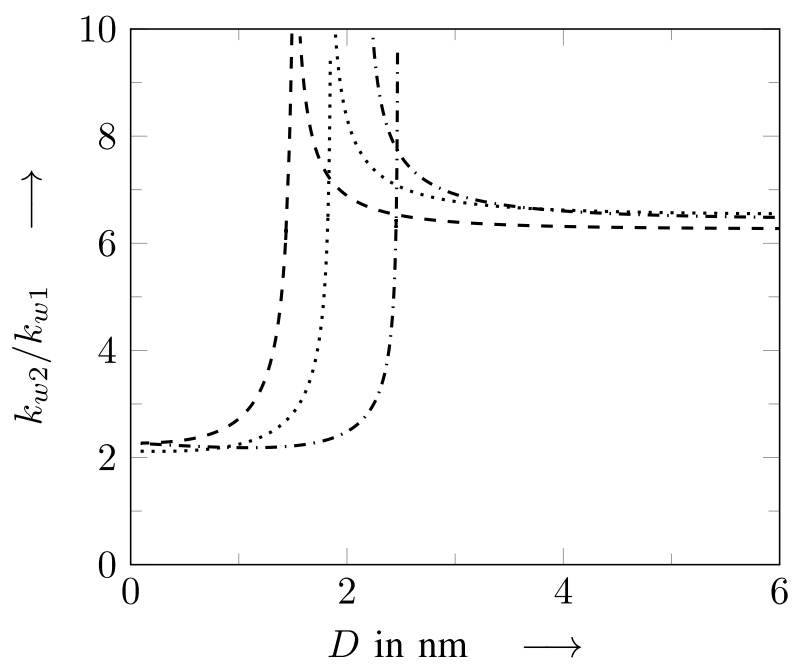

Fig. 10. Ratio of the first two eigenvalues; dotted line: $7.2 \mathrm{~N} / \mathrm{m}$; dash dotted line: $10 \mathrm{~N} / \mathrm{m}$; dashed line: $23.8 \mathrm{~N} / \mathrm{m}$.

Figure 10 shows the ratio of the first two eigenvalues. All intersections of the curve with integer values of the eigenvalue ratio indicate possible internal resonances. These resonances are mainly located close to bifurcation points. For an approach to the sample, internal resonances start with seven-to-one, whereas in retreat two-to-one internal resonances occur. Taking into account the influence of a variation of the beam's geometry, the values can be shifted to lower or higher internal resonances (see Figure 5).

\section{Dynamical Analysis}

In an SPM operation, each beam's amplitude is measured with the integrated sensor and is kept constant by applying a direct current $i_{\mathrm{dc}}$ to the actuator to set a static displacement and thus maintain a constant distance $D_{0}$ between each beam and a surface (c.f. Section III-A). The estimation of the resulting displacement as well as of the amplitude of oscillation for different combinations of $i_{\mathrm{dc}}$ and $i_{\mathrm{ac}}$ defines the accuracy and reliability of the process. To analyse the implications

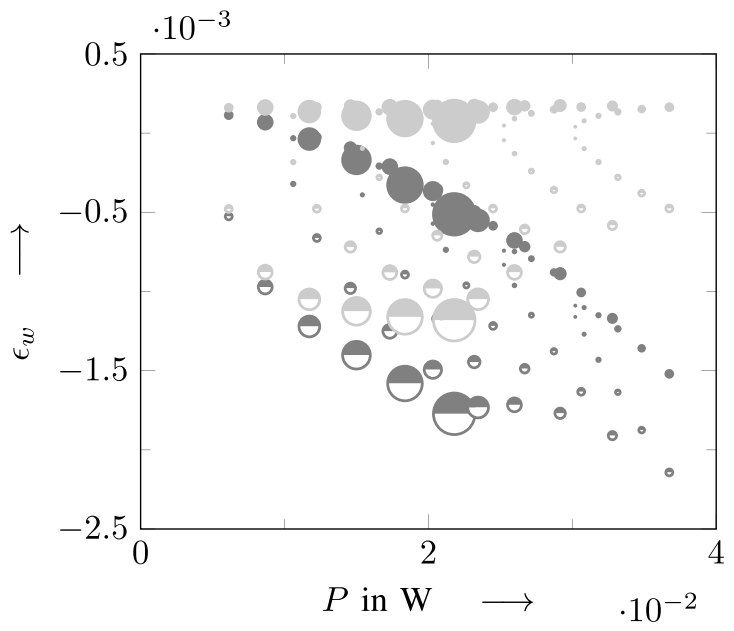

(a)

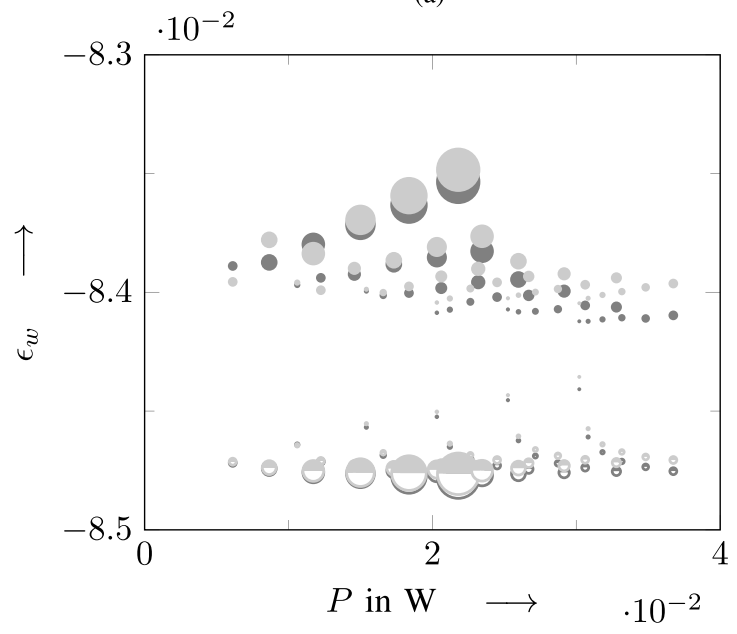

(b)

Fig. 11. Relative error of estimation with constant conversion factor; with $\left(\boldsymbol{J}_{7} \neq \boldsymbol{0}\right.$, full color $)$ and without $\left(\boldsymbol{J}_{7}=\boldsymbol{0}\right.$, light color) temperature dependent resistivity as well as with an excitation at $\Omega=\omega_{1}$ (full circle) and $\Omega=\omega_{1} / 2$ (half circle). (a) Static displacement, $\Omega=\omega_{1}$. (b) Amplitude, $\Omega=\omega_{1}$.

of the excitation on the systems behavior as well as on the estimation of the tip motion based on the sensor signal, (19) has been numerically integrated, using a variable order solver based on numerical differentiation formulas [31], for a variation of $i_{\mathrm{dc}}$ and $i_{\mathrm{ac}}$. The integration has been done until the system had reached steady state. Note that the system (19) has periodic coefficients, therefore the stability of the solutions has been investigated for the considered parameter domain by means of the absolute value of the characteristic multipliers (Floquet theory, [32]).

Figure 11 and Figure 12 show the resulting relative error of amplitude estimation over the power $P$ applied to the actuator, for an excitation at the first $\left(\Omega=\omega_{1}\right)$ and second $\left(\Omega=\omega_{2}\right)$ eigenfrequency of the system, respectively. The relative error is given by

$$
\epsilon_{\mathrm{w} n}=\frac{\hat{w}_{\mathrm{est} n}(\tilde{t})-\hat{w}_{n}\left(l_{t}, \tilde{t}\right)}{\hat{w}_{n}\left(l_{t}, \tilde{t}\right)},
$$

with the amplitude of the estimated displacement at the tip position $\hat{w}_{\mathrm{es} t n}=U_{\mathrm{b}} \kappa_{\mathrm{w} n}$, the actual amplitude of displacement 


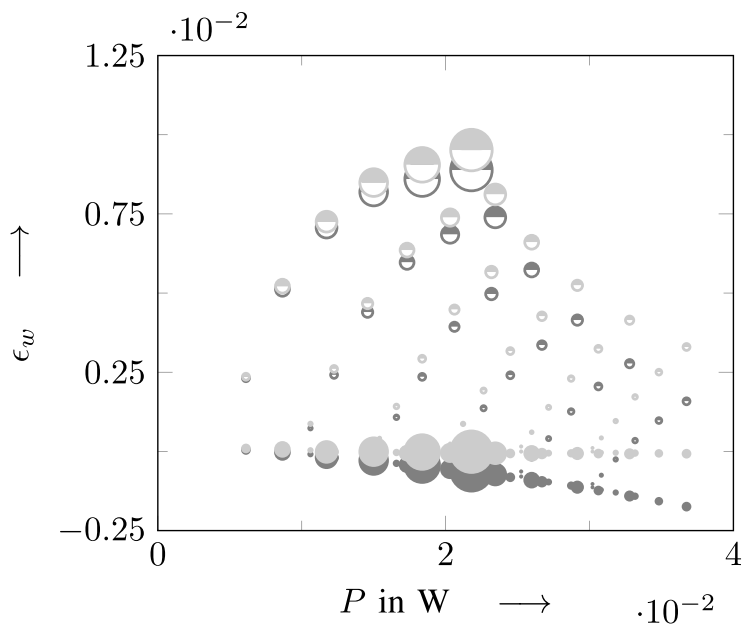

(a)

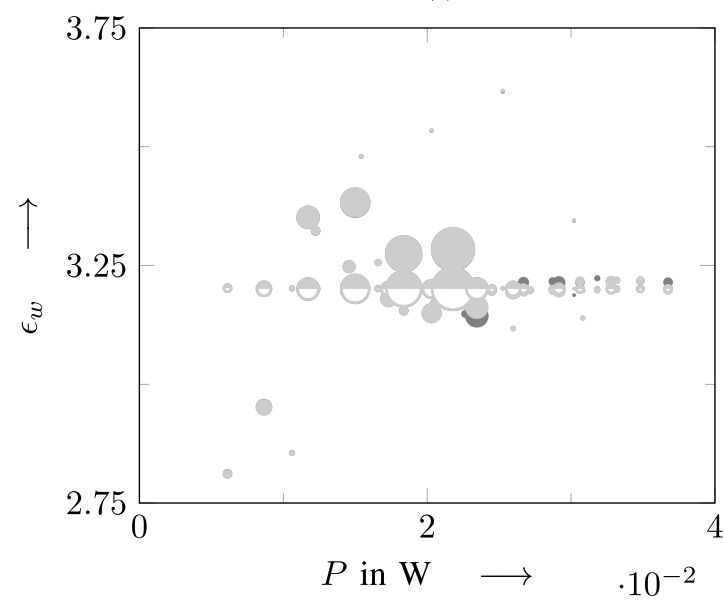

(b)

Fig. 12. Relative error of estimation with constant conversion factor; with $\left(\boldsymbol{J}_{7} \neq \boldsymbol{0}\right.$, full color) and without $\left(\boldsymbol{J}_{7}=\boldsymbol{0}\right.$, light color $)$ temperature dependent resistivity as well as with an excitation at $\Omega=\omega_{2}$ (full circle) and $\Omega=\omega_{2} / 2$ (half circle). (a) Static displacement, $\Omega=\omega_{2}$. (b) Amplitude, $\Omega=\omega_{2}$.

of the beam $\hat{w}_{n}$ and the conversion factor $\kappa_{\mathrm{w} n}$ (see (23)), each of mode $n$. The currents $i_{\mathrm{dc}}$ and $i_{\mathrm{ac}}$ are varied according to the parameters in Table I. The diameter of the markers in Figure 11 and Figure 12 represents the ratio between $i_{\mathrm{dc}}$ and $i_{\mathrm{ac}}$. Markers with a light color intensity have a temperature independent resistivity of the actuator $\left(\boldsymbol{J}_{7}=\boldsymbol{0}\right)$, full color intensity is the opposite $\left(\boldsymbol{J}_{7} \neq \boldsymbol{0}\right)$, half filled markers represent an excitation at half the resonance frequency $\left(\Omega=\omega_{n} / 2\right)$, full circles are for an excitation at the resonance frequency $\left(\Omega=\omega_{n}\right)$.

Figure 11 shows $\epsilon_{w}$ over $P$ for the static deflection and the amplitude at the first eigenfrequency. The relative error of the static displacement is increasing for an increasing power in case of an temperature dependent actuator. An excitation at half eigenfrequency also leads to an increasing error. For the amplitude at the first eigenfrequency, an excitation at half the frequency shows only a slight influence of the dissipated power. An excitation at the eigenfrequency exhibits an varying error for a variation in power. This is due to the square of the current in internal heat generation term (14), using half the frequency results in an $i_{\mathrm{dc}}$ independent excitation at twice the frequency, as can be seen in the following equation:

$$
\begin{aligned}
i^{2}= & \underbrace{i_{\mathrm{dc}}^{2}+\frac{i_{\mathrm{ac}}^{2}}{2}}_{\text {static excitation }}+\underbrace{2 i_{\mathrm{dc}} i_{\mathrm{ac}} \sin (\hat{\Omega} t)}_{\hat{\Omega} \text { excitation }} \\
& -\underbrace{\frac{i_{\mathrm{ac}}^{2} \cos (2 \hat{\Omega} t)}{2} .}_{2 \hat{\Omega} \text { excitation }} .
\end{aligned}
$$

The linear appearing change in the relative error for the $\Omega=\omega_{1}$ excitation is due to the variation of $i_{\mathrm{dc}}$, as can be seen from the increasing diameter of the markers. The slight increase of the error for an excitation at $\Omega=\omega_{1} / 2$ for a temperature dependent resistivity can be linked to the dissipated power within the actuator (14), since the temperature dependence of the aluminum layer leads to a coupling between excitation and temperature difference:

$$
g_{a j}(\tilde{t})=\underbrace{\frac{\rho_{e 0}}{A_{j 3}^{2}} i^{2}}_{\text {regular }}+\underbrace{\alpha_{e} \tilde{\theta} i^{2}}_{\text {coupling }}, \quad j=1,2
$$

Thus, the coupling term in (27) implies that the direct current $i_{\mathrm{dc}}$ also influences the response amplitude of the motion of $w(x, t)$ at $2 \hat{\Omega}$. The intensity of the coupling is defined by the electrical temperature coefficient of the aluminum layer as well as by the temperature difference itself.

Figure $12 \mathrm{a}$ and Figure $12 \mathrm{~b}$ show the same cantilever beam in case of an excitation at the second natural frequency. The behavior of the static error is similar to the case with an excitation at the first resonance frequency. An excitation at $\Omega=\omega_{1} / 2$ shows a stronger dependency of the error on the dissipated power. The amplitude error also exhibits an increased variation of the error in case of an excitation at $\Omega=\omega_{1}$ and an insignificant variation with an excitation at $\Omega=\omega_{1} / 2$. Compared to Figure 11 the error of the amplitude estimation is over an order of magnitude higher. This difference is due to the variation in slope of the eigenfunction at the sensor position. The conversion factor shows an increased sensitivity according to Figure 4. Depending on the actual geometry of the beam, the ratio between the conversion factor of the first mode to the conversion factor of the second mode varies between $\kappa_{\mathrm{w} 1} / \kappa_{\mathrm{w} 2}=5 . .20$.

\section{Summary AND CONClusion}

In this paper we have derived a nonlinear dynamical model for a microbeam with an electrothermal actuator and a piezoresistive sensor. This beam is designed as an element of an array of microbeams (see [33]), in which the single beams rely on an integrated sensing and actuation. The model incorporates the composite structure, a varying cross section as well as a thermal actuation and damping, based on an Euler-Bernoulli beam and Fourier heat conduction, respectively. It also yields the relation for the mechanical stress at the piezoresistive sensor elements, to analyse the voltage drop across a bridge circuit. The analysis is focused on the special properties of the microbeam (composite structure, varying cross section, thermal actuation, integrated sensor) and how they effect the modal, equilibria and dynamical behaviour of the system. 
Since the direct current is used for a distance control in SPM and SPL arrays, conclusions on the tip motion based on the integrated sensor are of special interest.

The modal analysis in Section III-A shows the dependency of the first three eigenmodes on the actuator geometry. Changes in eigenmode compared to an Euler-Bernoulli beam with a bulk structure mainly occur for higher modes and are located at the actuator position as well as at the free end of the beam (Figure 3). Since the sensor position is almost unaffected, the change in displacement between the modes can not be accounted for considering a constant conversion factor linking sensor voltage to tip displacement. Moreover, higher eigenmodes exhibit a steeper slope at the sensor position, leading to an increased mechanical stress. Thus, a tip motion estimation based on the sensor signal multiplied with a constant factor leads to an error when operating with higher modes or with multiple modes (see Figure 4). This error is due to the geometry of the beam and the sensor principle and it can be accounted for with a frequency dependent conversion factor. Due to an increased stress, the second mode offers an increased sensitivity in view of bridge voltage change due to a displacement of the microbeam. The modal analysis in Section III-A also shows a dependency of the ratio of two eigenfrequencies (e.g. $\omega_{2} / \omega_{1}$ ) on the geometrical parameters. With this dependency, experimentally obtained results presented in [11] can be linked to the composition of layers and actuator properties. Additionally, possible internal resonance can occur for specific configuration of the actuator.

The equilibrium analysis in Section III-B shows that internal resonances can be triggered to appear while approaching a sample surface. Depending on the composition of the beam and its sectional stiffness, the above mentioned resonances occur during an approach to a sample surface. To further shape this ratio, a nonideal support as well as change in sectional stiffness can be used (cf. [29]). Thus, a internal resonance can be adjusted to a certain distance between tip and sample and might be used for a control scheme or an additional measure or in case of multi frequency SPM, in which different properties are probed while scanning. Moreover, a variation of the tip sample interaction forces also results in an increasing deviation of the eigenmode, towards the free end of the beam. The highest deviation occurs for the first eigenmode and decreases for higher modes. The deviation of the eigenmode at the sensor position is negligible. Thus, this variation results in a conversion factor linking the displacement to sensor voltage, which varies with the tip sample distance, as can be seen in Figure 8. Especially for an array operation for SPL and SPM, the sensor signal needs to be interpreted correctly in order to set a defined distance as well as to gain the topography. Looking at the change of eigenvalues over tip sample distance (Figure 9) together with the increased sensitivity of the second eigenmode (Figure 4) shows that this increase is compensated by the decreased frequency to distance sensitivity.

A first dynamical analysis of the presented microbeam in Section III-C is focused on the estimation of the tip motion based on the integrated sensor. Using a constant conversion factor to gain the motion estimate from the sensor voltage, the error increases in case of an increased power dissipated within the actuator. Thus, changing the direct current leads to a change in excitation amplitude as well as an error in estimation of the tip motion, which leads to distortions and artifacts in an SPM and SPL array operation. The change in excitation amplitude due to a change in $i_{\mathrm{dc}}$ can also effect the amplitude of the microbeam at twice the excitation frequency. Cause for this effect is a temperature dependent resistivity of the thermal actuator's aluminum layer. Note that common practice is to assume the response amplitude at twice the excitation frequency to only depend on the alternating current $i_{\mathrm{ac}}$. Considering an excitation of the beam at its second resonance frequency, the estimation error increases by more than one order of magnitude. This effect can be linked to the different mode shapes of the system. Figure 3 shows the difference in slope at the sensor position for the first three modes and the difference in sensitivity of the sensor signal to beam displacement for the first two modes (Figure 4). Thus, the relation between the tip and the sensor position changes considering different modes for operation.

An application of the presented microbeam in array configuration to an SPM or SPL process leads to a misinterpretation of the sensor signal, when using a constant conversion factor. In contrast to an isotropic Euler Bernoulli beam model, the presented model shows a nonconstant ration between the conversion factor for the first two eigenmodes $(\kappa \mathrm{w} 2 / \kappa \mathrm{w} 1 \neq$ const). Thus, a calibration of the beams of an array only using the first eigenfrequency is not sufficient. As a result, a conversion factor for each mode should be used in order to minimize the error for tip motion estimation and increase the capability of the array to set constant distances for each individual beam.

\section{APPENDIX A \\ EIGENMODES AND - VALUES}

Within this sections the derivation of the comparison functions $\Phi_{w j n}$ and $\Phi_{\theta j n}$ is shown, which are used in Section II-C for the discretization of (19). These comparison functions satisfy all boundary and continuity conditions but do not satisfy the differential equations of (19). First, the following separation of variables has been introduced to the nondimensional form of (9) and (12)

$$
\begin{aligned}
w_{j}\left(x_{j}, t\right) & =\Phi_{w j}\left(x_{j}\right) q_{w}(t), \\
\theta_{j}\left(x_{j}, t\right) & =\Phi_{\theta j}\left(x_{j}\right) q_{\theta}(t),
\end{aligned}
$$

where $\Phi_{w j}$ and $\Phi_{\theta j}$ are spatial function and $q_{w}(t)$ and $q_{\theta}(t)$ are the corresponding time varying amplitudes. With the following simplifications

$$
\begin{gathered}
\Phi_{w}(x)= \begin{cases}\Phi_{w 1} ; & 0 \leq x<l_{1}, \\
\Phi_{w 2} ; & l_{1} \leq x<l_{2}, \\
\Phi_{w 3} ; & l_{2} \leq x<L,\end{cases} \\
\chi_{1 . .10}(x)= \begin{cases}\chi_{1 . .101} ; & 0 \leq x<l_{1}, \\
\chi_{1 . .102} ; & l_{1} \leq x<l_{2}, \\
\chi_{1 . .103} ; & l_{2} \leq x<L .\end{cases}
\end{gathered}
$$


the resulting equations is

$$
\begin{aligned}
\Phi_{w} \ddot{q}_{w}= & -\chi_{1} \Phi_{w} \dot{q}_{w}-\chi_{2} \Phi_{w}^{\prime \prime \prime \prime} q_{w} \\
& +\chi_{3} \Phi_{\theta}^{\prime \prime} q_{\theta}+\chi_{10} F_{\mathrm{TS}} \\
\Phi_{\theta} \dot{q}_{\theta}= & \left(\chi_{4} \Phi_{\theta}^{\prime \prime}+\chi_{6} \alpha_{e} \theta_{0} \Phi_{\theta} i^{2}\right) q_{\theta} \\
& -\chi_{5} \Phi_{w}^{\prime \prime} \dot{q}_{w}+\chi_{6} i^{2} \\
& +\chi_{9} U_{\mathrm{s}}^{2} .
\end{aligned}
$$

To approximate $\Phi_{w}$ and $\Phi_{\theta}$, the functions $U_{w k}$ and $U_{\theta k}$ are used with the following Ritz approach

$$
\begin{gathered}
\Phi_{w}=\sum_{k}^{\infty} a_{w k} U_{w k}(x)=\boldsymbol{a}_{w}^{T} \boldsymbol{U}_{w}, \\
\Phi_{\theta}=\sum_{k}^{\infty} a_{\theta k} U_{\theta k}(x)=\boldsymbol{a}_{\theta}^{T} \boldsymbol{U}_{\theta},
\end{gathered}
$$

where $a_{w k}$ and $a_{\theta k}$ are weighting constants of mode $k$. The functions $U_{w k}$ and $U_{\theta k}$ are eigenfunctions of a simplified system, which is homogeneous, undamped and with the interaction force being $F_{\mathrm{TS}}=0$. Thus, both equations satisfy all boundary and continuity conditions as well as the differential equations of the simplified system. Rearranging both equations (32) and (33) and multiply (32) with $\boldsymbol{U}_{w}$ and (33) with $\boldsymbol{U}_{\theta}$ as well as integrating both equations with respect to $x$ (Galerkin method) leads to the following set of ordinary differential equations, that governs the coupled motion of the microbeam.

$$
\begin{aligned}
\boldsymbol{J}_{1} \boldsymbol{a}_{w} \ddot{q}_{w}= & -\boldsymbol{J}_{2} \boldsymbol{a}_{w} \dot{q}_{w}-\boldsymbol{J}_{3} \boldsymbol{a}_{w} q_{w} \\
& +\boldsymbol{J}_{4} \boldsymbol{a}_{\theta} q_{\theta}+\boldsymbol{F}_{\mathrm{TS}}, \\
\boldsymbol{J}_{5} \boldsymbol{a}_{\theta} \dot{q}_{\theta}= & \left(\boldsymbol{J}_{6}+\boldsymbol{J}_{7} i^{2}\right) \boldsymbol{a}_{\theta} q_{\theta} \\
& -\boldsymbol{J}_{8} \boldsymbol{a}_{w} \dot{q}_{w}+\boldsymbol{J}_{9} i^{2}+\boldsymbol{J}_{10} U_{\mathrm{s}}^{2} .
\end{aligned}
$$

The constants $\boldsymbol{J}_{1.10}$ are used in analogy to the notation in Appendix B, whereas $\boldsymbol{\Phi}_{w}$ and $\boldsymbol{\Phi}_{\theta}$ are exchanged by $\boldsymbol{U}_{w}$ and $\boldsymbol{U}_{\theta}$, respectively. Introducing the isochronic approach $q_{w}=V_{w} \mathrm{e}^{\lambda t}$ and $q_{\theta}=V_{\theta} \mathrm{e}^{\lambda t}$ into (36) and (37), with $\overline{\boldsymbol{a}}_{w}=V_{w} \boldsymbol{a}_{w}$ and $\overline{\boldsymbol{a}}_{\theta}=V_{\theta} \boldsymbol{a}_{\theta}$, leads to the eigenvalue problem in case of $\boldsymbol{F}_{\mathrm{TS}}=\boldsymbol{0}$

$$
\begin{gathered}
\left(\boldsymbol{J}_{1} \lambda^{2}+\boldsymbol{J}_{2} \lambda+\boldsymbol{J}_{3}+\boldsymbol{J}_{4} \overline{\boldsymbol{a}}_{\theta}\right) \overline{\boldsymbol{a}}_{w}=0, \\
\overline{\boldsymbol{a}}_{\theta}=-\left(\boldsymbol{J}_{5} \lambda-\boldsymbol{J}_{6}\right)^{-1} \boldsymbol{J}_{8} \overline{\boldsymbol{a}}_{w} .
\end{gathered}
$$

Equations (38) and (39) also yield an approximation to the eigenfrequencies of (19) used in Section III-A as well as in Section III-B. For the latter, the influence of the interaction force has been included in (38) and (39) by

\begin{tabular}{|c|c|c|c|}
\hline Parameter & Symbol & Value & Unit \\
\hline Width & $B$ & $90 \cdot 10^{-6}$ & $\mathrm{~m}$ \\
\hline \multirow[t]{3}{*}{ Length } & $l_{1}$ & $30 \cdot 10^{-6}$ & $\mathrm{~m}$ \\
\hline & $l_{2}$ & $200 \cdot 10^{-6}$ & $\mathrm{~m}$ \\
\hline & $l_{3}$ & $10 \cdot 10^{-6}$ & $\mathrm{~m}$ \\
\hline \multirow[t]{3}{*}{ Hight } & $h_{1}$ & $3 \cdot 10^{-6}$ & $\mathrm{~m}$ \\
\hline & $h_{2}$ & $0.3 \cdot 10^{-6}$ & $\mathrm{~m}$ \\
\hline & $h_{3}$ & $1 \cdot 10^{-6}$ & $\mathrm{~m}$ \\
\hline \multirow[t]{2}{*}{ Heater width } & $b_{H 1}$ & $6 \cdot 10^{-6}$ & $\mathrm{~m}$ \\
\hline & $b_{H 2}$ & $4.5 \cdot 10^{-6}$ & $\mathrm{~m}$ \\
\hline Heater length & $l_{h}$ & $1.18 \cdot 10^{-3}$ & $\mathrm{~m}$ \\
\hline Tip Radii & $R_{t}$ & $20 \cdot 10^{-9}$ & $\mathrm{~m}$ \\
\hline \multirow[t]{3}{*}{ Young's modulus } & $E_{1}$ & $170 \cdot 10^{9}$ & $\mathrm{Nm}^{-2}$ \\
\hline & $E_{2}$ & $74 \cdot 10^{9}$ & $\mathrm{Nm}^{-2}$ \\
\hline & $E_{3}$ & $70 \cdot 10^{9}$ & $\mathrm{Nm}^{-2}$ \\
\hline \multirow[t]{2}{*}{ Possion's ratio } & $\nu_{t i p}$ & 0.22 & 1 \\
\hline & $\nu_{\text {sample }}$ & 0.22 & 1 \\
\hline \multirow[t]{3}{*}{ Density } & $\rho_{1}$ & 2300 & $\mathrm{kgm}^{-3}$ \\
\hline & $\rho_{2}$ & 2210 & $\mathrm{kgm}^{-3}$ \\
\hline & $\rho_{3}$ & 2694 & $\mathrm{kgm}^{-3}$ \\
\hline \multirow{3}{*}{$\begin{array}{l}\text { Coefficient of linear } \\
\text { thermal expansion }\end{array}$} & $\alpha_{1}$ & $2.616 \cdot 10^{-6}$ & $\mathrm{k}^{-1}$ \\
\hline & $\alpha_{2}$ & $0.54 \cdot 10^{-6}$ & $\mathrm{k}^{-1}$ \\
\hline & $\alpha_{3}$ & $23.3 \cdot 10^{-6}$ & $\mathrm{k}^{-1}$ \\
\hline \multirow[t]{3}{*}{ Specific heat capacity } & $c_{v j 1}$ & 713 & $\mathrm{~J}(\mathrm{kgK})^{-1}$ \\
\hline & $c_{v j 2}$ & 705 & $\mathrm{~J}(\mathrm{kgK})^{-1}$ \\
\hline & $c_{v j 3}$ & 753 & $\mathrm{~J}(\mathrm{kgK})^{-1}$ \\
\hline \multirow[t]{3}{*}{ Thermal conductivity } & $k_{t j 1}$ & 156 & $\mathrm{~W}(\mathrm{mK})^{-1}$ \\
\hline & $k_{t j 2}$ & 1.4 & $\mathrm{~W}(\mathrm{mK})^{-1}$ \\
\hline & $k_{t j 3}$ & 237 & $\mathrm{~W}(\mathrm{mK})^{-1}$ \\
\hline Heater resistance & $R_{h}$ & 24 & $\Omega$ \\
\hline Heater resistivity & $\rho_{e}$ & $2.857 \cdot 10^{-8}$ & $\Omega \mathrm{m}$ \\
\hline Sensor resistance & $R_{s}$ & 1090 & $\Omega$ \\
\hline Quality Factor & $Q$ & 516.13 & 1 \\
\hline Hamaker constant & $A_{H}$ & $0.425 \cdot 10^{-18}$ & $\mathbf{J}$ \\
\hline
\end{tabular}
a Taylor series expansion, considering only linear terms. Equations (34) and (35) set the basis for the analysis of the eigenmodes in Section III-A and III-B, to show the influence of a varied geometry and interaction forces on the modal behavior. Since (34) and (35) are developed including the first six terms, the functions $\Phi_{w}$ and $\Phi_{\theta}$ are considered comparison functions throughout the article.
TABLE I

PARAMETERS AND INTERVALS FOR VARIATION

\begin{tabular}{c|c} 
Constant & Variational range \\
\hline \hline$l_{2}$ & {$[0.2,0.8] \cdot L$} \\
$h_{3}$ & {$[0.05,0.25] \cdot H$} \\
$D$ & {$[0.1,10] \mathrm{nm}$} \\
$c_{v}$ & {$[0.5,1.5] \cdot c_{v}$} \\
$k_{t}$ & {$[0.5,1.5] \cdot k_{t}$} \\
$\alpha$ & {$[0.5,1.5] \cdot \alpha$} \\
$i_{\mathrm{dc}}$ & {$[5,30] \mathrm{mA}$} \\
$i_{\mathrm{ac}}$ & {$[5,30] \mathrm{mA}$}
\end{tabular}

TABLE II

Micro-Beam's Geometric and Material Parameter

\section{APPENDIX B}

\section{COEFFICIENTS}

The integrals of the discretization of (19)

$$
\begin{aligned}
\boldsymbol{J}_{1} & =\int_{(L)} \sum_{j} \boldsymbol{\Phi}_{w j} \boldsymbol{\Phi}_{w j}^{T} \mathrm{~d} x \quad \boldsymbol{J}_{2}=\int_{(L)} \sum_{j} \chi_{1 j} \boldsymbol{\Phi}_{w j} \boldsymbol{\Phi}_{w j}^{T} \mathrm{~d} x \\
\boldsymbol{J}_{3} & =\int_{(L)} \sum_{j} \chi_{2 j} \boldsymbol{\Phi}_{w j} \boldsymbol{\Phi}_{w j}^{\prime \prime \prime} T \mathrm{~d} x \quad \boldsymbol{J}_{4}=\int_{(L)} \sum_{j} \chi_{3 j} \boldsymbol{\Phi}_{w j} \boldsymbol{\Phi}_{\theta j}^{\prime \prime T} \mathrm{~d} x \\
\boldsymbol{J}_{5} & =\int_{(L)} \sum_{j} \boldsymbol{\Phi}_{\theta j} \boldsymbol{\Phi}_{\theta j}^{T} \mathrm{~d} x \quad \boldsymbol{J}_{6}=\int_{(L)} \sum_{j} \chi_{4 j} \boldsymbol{\Phi}_{\theta j} \boldsymbol{\Phi}_{\theta j}^{\prime \prime T} \mathrm{~d} x \\
\boldsymbol{J}_{7} & =\int_{(L)} \sum_{j} \chi_{6 j} \alpha_{e} \theta_{0} \boldsymbol{\Phi}_{\theta j} \boldsymbol{\Phi}_{\theta j}^{T} \mathrm{~d} x \quad \boldsymbol{J}_{8}=\int_{(L)} \sum_{j} \chi_{5 j} \boldsymbol{\Phi}_{\theta j} \boldsymbol{\Phi}_{w j}^{\prime \prime T} \mathrm{~d} x \\
\boldsymbol{J}_{9} & =\int_{(L)} \sum_{j} \chi_{6 j} \boldsymbol{\Phi}_{\theta j} \mathrm{~d} x \quad \boldsymbol{J}_{10}=\int_{\left(l_{1}\right)} \chi_{9 j} \boldsymbol{\Phi}_{\theta} \mathrm{d} x \\
\hat{\boldsymbol{\Phi}}_{w} & =\left[\begin{array}{ll}
\Phi_{w 31}\left(L_{t}\right) \ldots \Phi_{w 3 n}\left(L_{t}\right)
\end{array}\right]^{T}
\end{aligned}
$$


with the following coefficients:

$$
\begin{aligned}
& \chi_{j 1}=\frac{d_{j n}}{\mu_{j} \omega_{s}} \quad \chi_{2 j}=\frac{E_{1} \chi_{w 2 j}}{\mu_{j} \bar{A}_{j} L^{4} \omega_{s}} \\
& \chi_{3 j}=\frac{\chi_{w 1 j} E_{1} \theta_{0}}{\bar{A}_{j} \mu_{j} \omega_{s}^{2} H L^{2}} \quad \chi_{w 1 j}=\overline{A \alpha}_{j} \bar{S}_{y j}-{\overline{S_{y} \alpha}}_{j} \bar{A}_{j} \\
& \chi_{w 2 j}=\left(\bar{I}_{y j} \bar{A}-\bar{S}_{y j}^{2}\right) \quad \chi_{4 j}=\frac{{\overline{k_{t} A}}_{j}}{\chi_{\theta 1 j} L^{2} \omega_{s}} \\
& \chi_{5 j}=\frac{T_{0} E_{1} H \chi_{\theta 2 j}}{\chi_{\theta 1 j} L^{2} \theta_{0}} \quad \chi_{6 j}=\frac{\bar{A}_{j} \rho_{e 0}}{\chi_{\theta 1 j} \theta_{0} \omega_{s} A_{j 3}^{2}} \\
& \chi_{\theta 1 j}={\overline{c_{0} \rho A}}_{j}+T_{0} E_{1} \overline{A \alpha}_{j}^{2} \bar{A}_{j}^{-1} \quad \chi_{\theta 2 j}=\bar{S}_{y j} \overline{A \alpha}_{j} \bar{A}_{j}^{-1}-\bar{S}_{y}{ }_{j} \\
& \chi_{7}=\frac{A_{h} R_{t} \hat{\boldsymbol{\Phi}}_{w} \sigma^{6}}{180 H \omega_{s}^{2} \mu L} \quad \chi_{8}=\frac{A_{h} R_{t} \hat{\boldsymbol{\Phi}}_{w}}{6 H \omega_{s}^{2} \mu L} \\
& \chi_{9 j}=\frac{\bar{A}_{1}}{\chi_{\theta 1} \theta_{0} \omega_{S} R_{S} V_{S}} \\
& \chi_{10 j}=\frac{1}{\bar{A}_{j} \mu_{j} \omega_{s}^{2} H} \\
& \chi_{b c 1 j}=\chi_{w 2 j} \frac{E_{1} H}{\bar{A}_{j} L^{2}} \quad \chi_{b c 2 j}=\chi_{w 1 j} \frac{E_{1} \theta_{0}}{\bar{A}_{j}} \\
& I=i_{d c}+i_{a c} \sin (\hat{\Omega} t) \quad \hat{\Omega}=\frac{\Omega}{\omega_{s}} \\
& l_{h j}=l_{j} N_{j} \quad \tilde{P}=\frac{\rho_{e} l_{h}}{A_{3 j}}\left(1+\alpha_{e} \theta_{0} \theta_{j}\right) I^{2} \\
& L_{t}=\frac{L-l_{t}}{L} \quad \xi_{0}=D-h_{t}
\end{aligned}
$$

\section{APPENDIX C}

\section{PARAMETERS USED FOR SIMULATIONS}

See Tables I and II.

\section{REFERENCES}

[1] M. Kaestner, M. Hofer, and I. W. Rangelow, "Nanolithography by scanning probes on calixarene molecular glass resist using mix-andmatch lithography," J. Micro/Nanolithogr., MEMS MOEMS, vol. 12, no. 3, pp. 031111-1-031111-13, 2013.

[2] N. Jalili and K. Laxminarayana, "A review of atomic force microscopy imaging systems: Application to molecular metrology and biological sciences," Mechatronics, vol. 14, no. 8, pp. 907-945, Oct. 2004.

[3] B. Bhushan, H. Fuchs, and M. Tomitori, Eds., Applied Scanning Probe Methods VIII: Scanning Probe Microscopy Techniques. New York, NY, USA: Springer, 2008.

[4] M. Kaestner et al., "Scanning probes in nanostructure fabrication," J. Vac. Sci. Technol. B, vol. 32, no. 6, p. 06F101, 2014.

[5] Y. Sarov, T. Ivanov, A. Frank, and I. W. Rangelow, "Thermally driven multi-layer actuator for 2D cantilever arrays," Appl. Phys. A, Mater. Sci. Process., vol. 102, pp. 61-68, Jan. 2010.

[6] G. E. Fantner et al., "DMCMN: In depth characterization and control of AFM cantilevers with integrated sensing and actuation," J. Dyn. Syst., Meas., Control, vol. 131, no. 6, p. 061104, Nov. 2009.

[7] Y. Sarov, T. Ivanov, A. Frank, and I. W. Rangelow, "Controllable off-plane deflection of cantilevers for multiple scanning proximity probe arrays," Appl. Phys. A, vol. 92, no. 3, pp. 525-530, Aug. 2008.

[8] R. Pedrak et al., "Micromachined atomic force microscopy sensor with integrated piezoresistive sensor and thermal bimorph actuator for highspeed tapping-mode atomic force microscopy phase-imaging in higher eigenmodes," J. Vac. Sci. Technol. B, vol. 21, no. 6, pp. 3102-3107, 2003.

[9] K. Ivanova et al., "Scanning proximal probes for parallel imaging and lithography," J. Vac. Sci. Technol., vol. 26, no. 6, pp. 2367-2373, 2008.

[10] T. Ivanov, "Piezoresistive cantilevers with an integrated bimorph actuator,' Ph.D. dissertation, Dept. Phys., Univ. Kassel, Kassel, Germany, 2004.
[11] M. Woszczyna et al., "High eigenmode operation of cantilevers with integrated piezoresistive readout and bimetal actuator," in Pronano Proceedings. Münster, Germany: Monsenstein und Vannerdat, 2010, pp. 243-253.

[12] T. Sulzbach et al., "Individually addressable cantilever arrays with integrated sharp tips for parallel surface scanning," in Pronano Proceedings. Münster, Germany: Monsenstein und Vannerdat, 2010, pp. 139-149.

[13] A. Frank et al., "Suppression of cross-talk effects of self actuated piezoresistive SPM-cantilever arrays," in Pronano Proceedings. Münster, Germany: Monsenstein und Vannerdat, 2010, pp. 169-178.

[14] D. Bullen, S.-W. Chung, X. Wang, J. Zou, C. A. Mirkin, and C. Liu, "Parallel dip-pen nanolithography with arrays of individually addressable cantilevers," Appl. Phys. Lett., vol. 84, no. 5, pp. 789-791, 2004.

[15] N. Watanabe, Y. Isono, T. Kakinaga, T. Nagamura, and T. Sasaki, "Thermal actuated multi-probes cantilever array for scanning probe parallel nano writing system," in Proc. MEMS, Kobe, Japan, Jan. 2007, pp. 99-102.

[16] R. Li, Q.-A. Huang, and W. Li, "A nodal analysis model for the outof-plane beamshape electrothermal microactuator," Microsyst. Technol., vol. 15, no. 2, pp. 217-225, Feb. 2009.

[17] F. L. Guo and G. A. Rogerson, "Thermoelastic coupling effect on a micro-machined beam resonator," Mech. Res. Commun., vol. 30, no. 6, pp. 513-518, Nov.Dec. 2003.

[18] Y. Sun, D. Fang, and A. K. Soh, "Thermoelastic damping in microbeam resonators," Int. J. Solids Struct., vol. 43, no. 10, pp. 3213-3229, May 2006.

[19] R. Lifshitz and M. L. Roukes, "Thermoelastic damping in microand nanomechanical systems," Phys. Rev. B, vol. 61, pp. 5600-5609, Feb. 2000.

[20] R. Lifshitz and M. C. Cross, "Nonlinear dynamics of nanomechanical resonators," in Nonlinear Dynamics of Nanosystems. Hoboken, NJ, USA: Wiley, Mar. 2008

[21] R. B. Hetnarski and M. R. Eslami, Thermal Stresses: Advanced Theory and Applications, vol. 158. New York, NY, USA: Springer, 2008.

[22] E. Pestel and J. Wittenburg, Technische Mechanik Band 2: Festigkeitslehre. Mannheim/Leipzig/Wien/Zrich: BI-Wiss.-Verl., 1992.

[23] Y. C. Fung and P. Tong, Classical and Computational Solid Mechanics, vol. 1. Singapore: World Scientific, 2001.

[24] P. Hagedorn and A. DasGupta, Vibrations and Waves in Continuous Mechanical Systems. Hoboken, NJ, USA: Wiley, 2007.

[25] D. Sarid, T. G. Ruskell, R. K. Workman, and D. Chen, "Driven nonlinear atomic force microscopy cantilevers: From noncontact to tapping modes of operation," J. Vac. Sci. Technol. B, Microelectron. Nanometer Struct., vol. 14, no. 2, pp. 864-867, 1996.

[26] J. N. Israelachvili, Intermolecular and Surface Forces: Revised, 3rd ed. San Francisco, CA, USA: Academic, 2011.

[27] C.-L. Tien, A. Majumdar, and F. Gerner, Microscale Energy Transport. Boca Raton, FL, USA: CRC Press, 1998.

[28] R. P. Brent, "An algorithm with guaranteed convergence for finding a zero of a function," Comput. J., vol. 14, no. 4, pp. 422-425, 1971.

[29] E. Hacker and O. Gottlieb, "Internal resonance based sensing in noncontact atomic force microscopy," Appl. Phys. Lett., vol. 101, no. 5, pp. 053106-1-053106-5, 2012.

[30] Y. Kuznetsov, Elements of Applied Bifurcation Theory, vol. 112. New York, NY, USA: Springer, 1998.

[31] L. F. Shampine and M. W. Reichelt, "The MATLAB ODE suite," SIAM J. Sci. Comput., vol. 18, no. 1, pp. 1-22, 1997.

[32] L. Meirovitch, Methods of Analytical Dynamics. New York, NY, USA: McGraw-Hill, 1970

[33] T. Sulzbach and I. W. Rangelow, Eds., PRONANO: Proceedings of the Integrated Project on Massively Parallel Intelligent Cantilever Probe Platforms for Nanoscale Analysis and Synthesis. Münster, Germany: Monsenstein und Vannerdat, 2010.

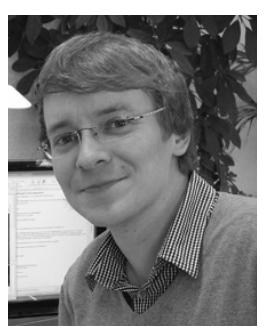

Dennis Roeser recevied the Diplome degree from the University of Paderborn, in 2010. He is currently pursuing the $\mathrm{Ph} . \mathrm{D}$. degree with the Ilmenau University of Technology based on the Carl Zeiss Foundation Scholarship. He is a Scientific Staff Member on modeling, analysis, and control of MEMS arrays. His research interests are nonlinear dynamics and coupling phenomena of arrays, the utilization of nonlinear dynamics for control and performance enhancements, and systems with integrated actuation and sensing, all in the area 


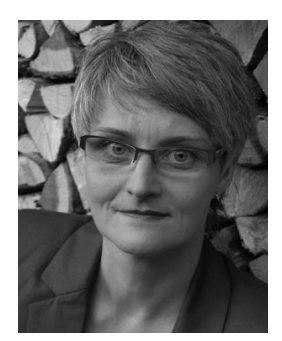

Stefanie Gutschmidt received the Ph.D. degree in applied mechanics from the Darmstadt University of Technology, Darmstadt, Germany, in 2005. She was a Postdoctoral Research Fellow with the Technion-Israel Institute of Technology, Haifa, Israel, (2005-2008), and the University of Liege, Liege, Belgium (2008). Since 2009, she has been a Senior Lecturer with the Department of Mechanical Engineering, University of Canterbury, Christchurch, New Zealand. Her current research interests are nonlinear dynamics and vibrations, MEMS and NEMS technology, mechatronics, earthquake engineering, structural health monitoring using wireless smart sensors, and energy harvesting based on structural vibration.

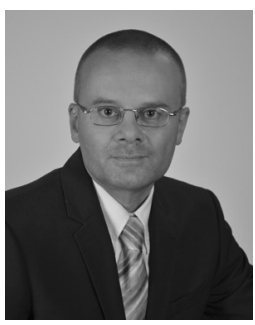

Thomas Sattel received the Diploma degree in mechanical engineering, the Pre-Diploma degree in mathematics, and the Ph.D. degree in mechanical engineering from the Darmstadt University of Technology, Germany, in 1995, 1996, and 2002, respectively. In 2008, he was a Lecturer and an Assistant Professor of Mechatronics with the University of Paderborn, Germany. He is currently a Professor with the Department of Mechanical Engineering, Ilmenau University of Technology, and the Head of the Mechatronics Group. In 2009, he was a co-recipient of the German Innovation Award in Medical Technology from the Federal Ministry of Education and Research. His research interests include dynamics and vibrations of mechatronic systems and MEMS, and novel integrated electromechanical actuators and motors for medical applications, manufacturing, automotive, robotic, precision engineering, and power engineering devices.

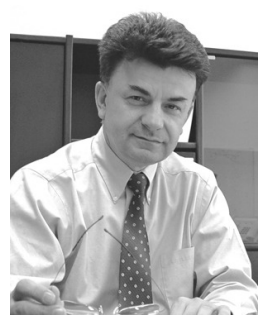

Ivo W. Rangelow received the M.S. and $\mathrm{Ph} . \mathrm{D}$. degrees in electronics from the University of Wroclaw, in 1979 and 1983, respectively. From 1983 to 1984, he was with the University of Muenster, where his research focused on the development of ion and electron beam techniques. In 1985, he joined the Fraunhofer-Institute, Berlin, where he was involved in X-ray lithography. In 1986, he joined the University of Kassel, where he focused on the development of small cantilever sensors, microresonators, diverse MEMS, and novel gas-chopping techniques for plasma etching of silicon for high aspect ratio devices. In 2005, he joined the Ilmenau University of Technology and his actual works are focused on sub-10 nm scanning probe-based lithography. He was a Guest Professor with the University of Vienna, Wroclaw University, and the University of Berkeley. He is currently the Director of the Institute of Micro- and Nanoelectronics with the Ilmenau University of Technology. $\mathrm{He}$ has authored or co-authored over 300 scientific papers and holds 48 patents. 